\title{
ERG induces androgen receptor-mediated regulation of SOX9 in prostate cancer
}

\author{
Changmeng Cai, ${ }^{1}$ Hongyun Wang, ${ }^{1}$ Housheng Hansen He, ${ }^{2,3}$ Sen Chen, ${ }^{1}$ Lingfeng He, ${ }^{1}$ Fen Ma, ${ }^{1}$ \\ Lorelei Mucci, ${ }^{4}$ Qianben Wang, ${ }^{2}$ Christopher Fiore, ${ }^{2}$ Adam G. Sowalsky, ${ }^{1}$ Massimo Loda, ${ }^{2}$ \\ X. Shirley Liu, ${ }^{3}$ Myles Brown, ${ }^{2}$ Steven P. Balk, ${ }^{1}$ and Xin Yuan ${ }^{1}$
}

\begin{abstract}
${ }^{1}$ Hematology-Oncology Division, Department of Medicine, Beth Israel Deaconess Medical Center and Harvard Medical School, Boston, Massachusetts, USA. ${ }^{2}$ Department of Medical Oncology and ${ }^{3}$ Department of Biostatistics and Computational Biology, Dana Farber Cancer Institute and Harvard Medical School, Boston, Massachusetts, USA. ${ }^{4}$ Departments of Epidemiology and Nutrition, Dana Farber Cancer Institute and Harvard School of Public Health, Boston, Massachusetts, USA.
\end{abstract}

\begin{abstract}
Fusion of the androgen receptor-regulated (AR-regulated) TMPRSS2 gene with ERG in prostate cancer (PCa) causes androgen-stimulated overexpression of ERG, an ETS transcription factor, but critical downstream effectors of ERG-mediating PCa development remain to be established. Expression of the SOX9 transcription factor correlated with TMPRSS2:ERG fusion in 3 independent PCa cohorts, and ERG-dependent expression of SOX9 was confirmed by RNAi in the fusion-positive VCaP cell line. SOX9 has been shown to mediate ductal morphogenesis in fetal prostate and maintain stem/progenitor cell pools in multiple adult tissues, and has also been linked to $\mathrm{PCa}$ and other cancers. SOX9 overexpression resulted in neoplasia in murine prostate and stimulated tumor invasion, similarly to ERG. Moreover, SOX9 depletion in VCaP cells markedly impaired invasion and growth in vitro and in vivo, establishing SOX9 as a critical downstream effector of ERG. Finally, we found that ERG regulated SOX9 indirectly by opening a cryptic AR-regulated enhancer in the SOX9 gene. Together, these results demonstrate that ERG redirects AR to a set of genes including SOX9 that are not normally androgen stimulated, and identify SOX9 as a critical downstream effector of ERG in TMPRSS2:ERG fusion-positive PCa.
\end{abstract}

\section{Introduction}

The androgen receptor (AR) plays a central role in prostate cancer (PCa) development, and its transcriptional functions are partially or fully restored in the tumors that relapse after androgen deprivation therapy (castration-resistant prostate cancer, CRPC) (1). The role of AR in PCa was further strengthened by the discovery of recurrent genomic rearrangements that result in AR-driven overexpression of ETS family transcription factor proto-oncogenes, and in particular the v-ets erythroblastosis virus E26 oncogene homolog, ERG. In approximately half of primary PCa cases, a gene fusion between the $5^{\prime}$ untranslated region of the androgen-regulated TMPRSS 2 gene and an exon in the ERG gene results in androgen-regulated high-level expression of a transcriptionally active, $\mathrm{N}$-terminal-truncated ERG protein (amino acids 1-44 being deleted in the most common fusion) $(2,3)$. This fusion is an early event, as it is found in precursor prostatic intraepithelial neoplasia (PIN) lesions located adjacent to TMPRSS2:ERG fusion-positive cancers (4). The fusion gene is also highly expressed in CRPC, indicating that overexpressed ERG contributes to PCa development and progression (5).

The importance of ERG is further supported by studies in the TMPRSS2:ERG fusion-positive VCaP cell line, where RNAi-mediated downregulation of ERG impairs cell growth and invasion (6, 7). Moreover, transgenic overexpression of ERG in mouse prostate causes increased proliferation, and in combination with the loss of one PTEN allele, results in PIN or invasive PCa (8-11). Several

Authorship note: Changmeng Cai and Hongyun Wang contributed equally to this work.

Conflict of interest: The authors have declared that no conflict of interest exists. Citation for this article: J Clin Invest. 2013;123(3):1109-1122. doi:10.1172/JCI66666.
ERG-regulated genes in TMPRSS2:ERG fusion-positive PCa cases have been identified, with EZH2 being one such candidate ERG target gene $(6,7,11-15)$. Additionally, a recent study found that there was marked overlap between ERG and AR binding sites, and that ERG repressed both AR expression and AR activity on these sites, suggesting that downregulation of AR activity on subsets of genes may be a mechanism of ERG action (12). However, the downstream effectors and functions of ERG that drive PCa remain to be firmly established.

SOX9 belongs to the SOX ( $\underline{S} R Y$-related HMG box) family of transcription factors and regulates many developmental processes $(16,17)$. SOX9 mutations cause the disease campomelic dysplasia, which is characterized by extreme cartilage and bone malformation, frequent XY sex reversal, and multiple defects in other organs, consistent with an important role in tissue development $(18,19)$. In the adult, SOX9 contributes to maintenance of stem/progenitor cells in tissues including intestine, liver, pancreas, and hair follicle (20-24), and dysregulated SOX9 expression has been implicated in the pathogenesis of several cancers (25-31). Similar to AR, SOX9 is required for prostate development, and accumulating evidence indicates that it contributes to the development of PCa (32-39).

SOX9 in developing prostate is expressed by epithelial cells invading into urogenital sinus mesenchyme, and loss of SOX9 causes a profound defect in prostate ductal morphogenesis (33, $35,36,39)$. In adult human prostate, SOX9 is normally expressed primarily by the basal cells, which surround the strongly AR-positive luminal epithelium and express only low levels of AR (34). In contrast, SOX9 and AR are coexpressed in PCa cells, with an increased fraction of SOX9-positive cells being found in CRPC 
A

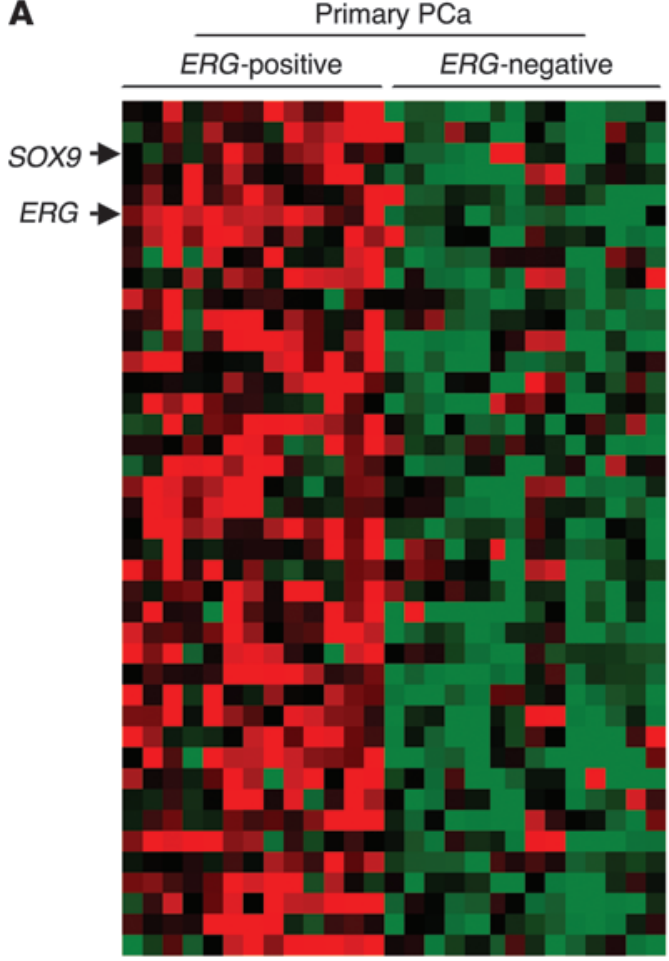

B

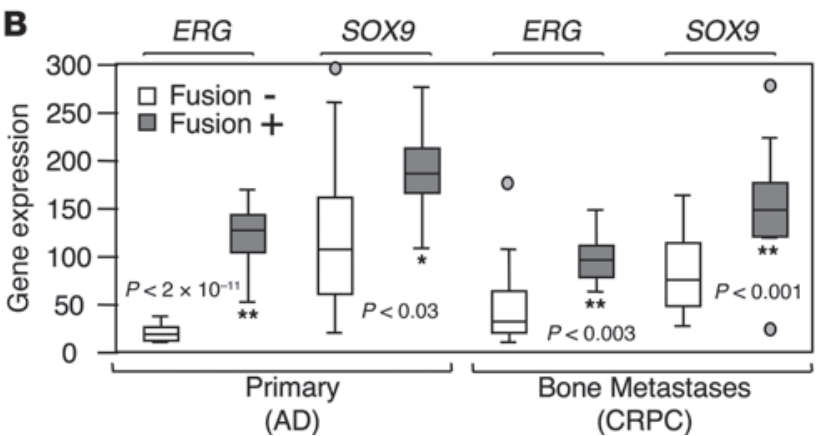

Metastatic CRPC
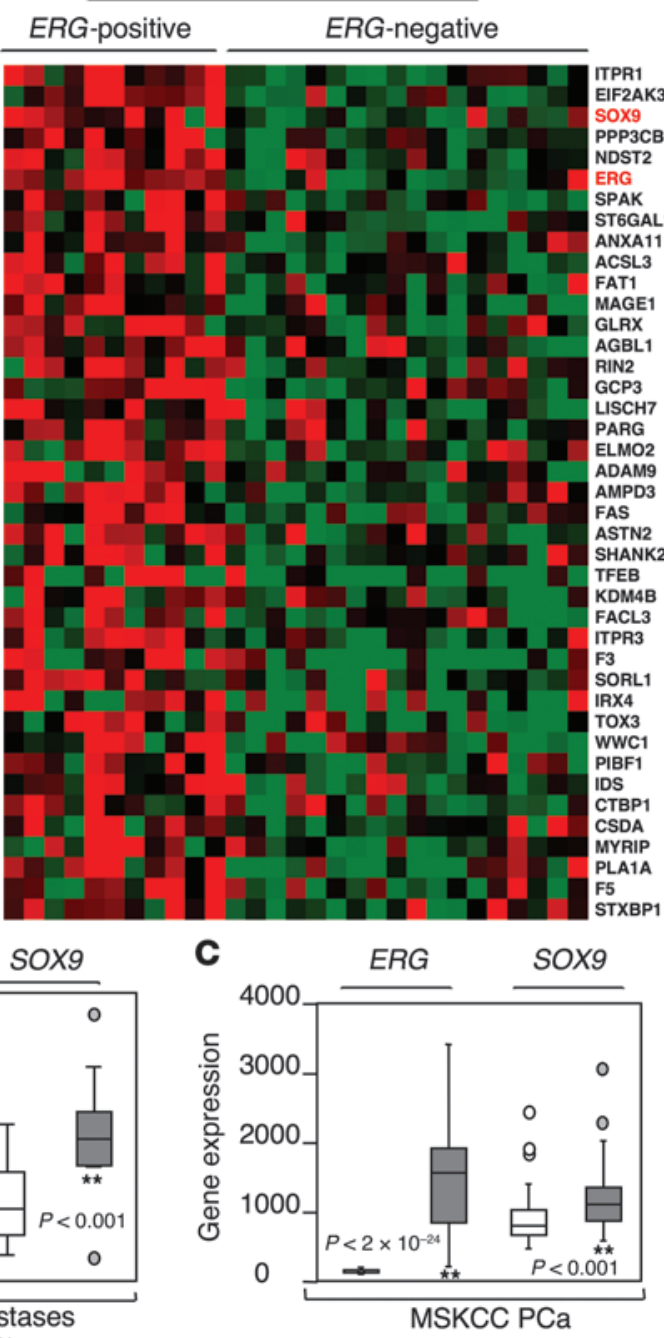

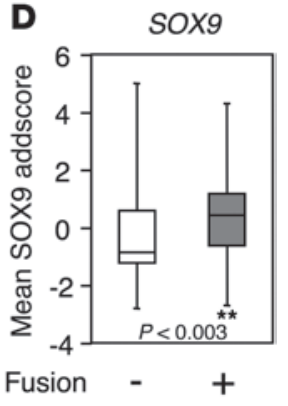

Figure 1

SOX9 expression is increased in TMPRSS2:ERG-positive PCa. (A) Heat maps (green, lower expression; red, higher expression) of Affymetrix microarray data showing mRNA expression for genes with increased levels in TMPRSS2:ERG fusion-positive versus TMPRSS2:ERG fusionnegative tumor subsets of both primary PCa and CRPC bone metastases (fold change of mean expression $>1.5$ and a 2-tailed Student's $t$ test $P$ value < 0.05). (B) Quartile plot (mean and each quartile) of ERG and SOX9 expression. The differences between the fusion-positive and fusion-negative samples were ${ }^{\star} P<0.05$ or ${ }^{\star \star} P<0.01$ (2-tailed Student's $t$ test). (C) MSKCC dataset showing that SOX9 expression was significantly higher in ERG-positive tumors (based on the outlier expression of ERG, 47/103) versus ERG-negative tumors. Heat maps for ERG and SOX9 expression in individual tumors are shown in Supplemental Figure 1A. (D) SOX9 expression index based on SOX9 immunostaining intensity and fraction of positive cells (mean SOX9 addscore) are plotted for TMPRSS2:ERG fusion-positive versus -negative radical prostatectomy samples from the Physicians' Health and Health Professionals Follow-up Studies.

and correlating with more aggressive behavior $(32,34)$. Moreover, a 17q24.3 SNP associated with PCa has been functionally mapped to an enhancer of the SOX9 gene, and allele-specific recruitment of transcription factors to this enhancer can increase SOX9 and may mediate the increased PCa risk (38). Silencing endogenous SOX9 expression in $\mathrm{PCa}$ xenografts by shRNA causes reduced tumor growth, while SOX9 overexpression enhances PCa xenograft growth and invasion (35). Finally, similarly to ERG, transgenic overexpression of SOX9 in mouse prostate epithelium causes hyperplasia, and on a Pten-deficient background, can enhance PIN development (32). We report here that SOX9 is a critical down- stream effector of ERG in TMPRSS2:ERG fusion-positive PCa, and that ERG stimulates SOX9 expression by redirecting AR to a cryptic AR-regulated enhancer in the $\mathrm{SOX} 9$ gene.

\section{Results}

SOX9 expression is correlated with TMPRSS2:ERG fusion in primary and metastatic CRPC. We reported previously that expression of the AR-regulated TMPRSS2:ERG fusion gene is restored in CRPC, consistent with the reactivation of AR transcriptional activity and a continued important role for ERG in CRPC (5). Therefore, to identify genes that may be regulated by ERG in vivo, we 
examined our previously reported PCa gene expression dataset (40) for genes that were increased in TMPRSS2:ERG-positive primary PCa and metastatic CRPC (Figure 1A). SOX9 was among the genes that were most significantly correlated with TMPRSS2:ERG fusion in both the primary and metastatic CRPC tumors (Figure 1, A and B). To confirm this correlation between SOX9 and TMPRSS2:ERG fusion, we next examined a recently reported large primary PCa dataset from MSKCC (41), and again found that SOX9 expression was significantly correlated with TMPRSS2:ERG fusion (Figure 1C; heat map shown in Supplemental Figure $1 \mathrm{~A}$; supplemental material available online with this article; doi:10.1172/JCI66666DS1).

To determine whether SOX9 protein expression also correlated with TMPRSS2:ERG fusion, we carried out SOX9 immunohistochemical (IHC) assessment on a primary PCa tissue microarray from the Physicians' Health and Health Professionals Follow-up Studies, which had been assessed previously for TMPRS$S 2: E R G$ fusion by FISH and IHC $(42,43)$. Each tumor was scored based on staining intensity and fraction of cells that were positive (SOX9 addscore; representative SOX9-positive and -negative staining is shown in Supplemental Figure 1B). This analysis again showed a significant correlation between TMPRSS2:ERG fusion and SOX9 expression (Figure 1D). Together, these results indicated that SOX9 may be directly or indirectly regulated by ERG in TMPRSS2:ERG-positive PCa. Previous reports have established critical roles for SOX9 during prostate development and in stem/ progenitor cell maintenance of multiple tissues, as well as in the pathogenesis of several cancers including PCa $(20-35,37,38)$. Therefore, we focused on SOX9 as a possible effector of ERG in TMPRSS2:ERG fusion-positive PCa.

ERG regulates SOX9 expression in TMPRSS2:ERG fusion-positive PCa cells. We next compared available PCa cell lines for SOX9 expression. Significantly, the basal SOX9 mRNA level was higher in the VCaP cell line (which is the only available TMPRSS2:ERG fusion-positive PCa cell line) relative to the SOX9 levels in a series of fusion-negative PCa cell lines (Figure 2A). Moreover, the DHTstimulated increase in ERG mRNA was associated with a marked increase in SOX9 mRNA in the VCaP cells (Figure 2, A and B). Interestingly, the relatively low basal level of SOX9 in LNCaP cells was not increased by DHT, and was instead moderately repressed (Figure 2, A and B). These apparent cell-specific responses of SOX9 to DHT correlated with binding of phospho-RNA polymerase II to the SOX9 promoter, indicating that they were transcriptional (Figure 2C). At the protein level, SOX9 in VCaP cells also increased in response to DHT in a dose-dependent fashion, similarly to the androgen-regulated PSA and ERG proteins in these cells, and this was blocked by an AR antagonist, bicalutamide (Bic) (Figure 2D, and data not shown). In contrast, SOX9 protein was decreased by DHT in LNCaP cells (Figure 2E).

We next examined VCaP xenografts to determine whether there was a correlation in vivo between SOX9 and ERG. We showed previously that AR and ERG were highly expressed in these xenografts prior to castration, declined markedly immediately after castration, and were again highly expressed in the relapsed castration-resistant xenografts (5). As shown in Figure 2F, SOX9 protein showed a similar marked decrease after castration and was then highly expressed again in the relapsed castration-resistant xenograft. Consistent with the IHC assessment, ERG and SOX9 mRNA levels declined immediately after castration and were restored in the relapsed castration-resistant xenografts (Sup- plemental Figure 2A). Finally, we used RNAi to directly examine whether ERG was regulating SOX9 expression. We generated stable lentiviral shERG-infected VCaP cells and found that basal- and DHT-stimulated SOX9 mRNA and protein expression were markedly decreased (Figure 2, G and $\mathrm{H}$ ). We obtained similar results using another ERG shRNA (shERG-2, Supplemental Figure 2B) and by transient transfection with an independent ERG siRNA (Supplemental Figure 6B). Although additional mechanisms may further modulate the expression of SOX9 in fusion-negative or -positive tumors (34), these data establish that SOX9 is increased by ERG in TMPRSS2:ERG fusion-positive PCa.

SOX9 is a critical downstream effector of ERG in TMPRSS2:ERG fusion-positive PCa. The ERG regulation of SOX9, in conjunction with accumulating data indicating that SOX9 contributes to oncogenesis, indicated that SOX9 may be an important downstream effector of ERG in TMPRSS2:ERG-fusion PCa. Therefore, we next focused on whether SOX9 could mediate functions associated with ERG in PCa cells. Previous studies had shown that ERG expression in VCaP cells enhanced cell invasion $(6,7)$, while transgenic overexpression of ERG in murine prostate was found to stimulate hyperplasia, dysplasia, or PIN, and to drive progression to PCa when expressed on a Pten ${ }^{+/-}$background $(8,9)$. Similar to the in vitro effects of ERG, we reported previously that overexpression of SOX9 in LNCaP xenografts caused increased proliferation and invasion (35). Moreover, a recent study found that transgenic overexpression of SOX9 caused hyperplasia and could cooperate with PTEN loss to stimulate tumor formation (32).

Consistent with this latter report, we found that transgenic prostate epithelial overexpression of Flag epitope-tagged SOX9 resulted in PIN lesions in a fraction of mice ( 4 of 9) analyzed at 5 to 8 months of age. Although the $S O X 9$ transgene was expressed in all prostate lobes (Supplemental Figure 3A), the PIN lesions were primarily in the ventral lobe (Figure 3, SOX9). We also observed PIN in a fraction of mice (5 of 9) that were $\mathrm{Pten}^{+/}$, although these lesions were more prominent in the dorsolateral and anterior lobes, and spared the ventral lobe (Figure 3, $P T E N^{+/-}$, and Supplemental Figure 3B). In contrast, all compound $P T E N^{+/-} ; S O X 9$ mice (19 of 19) developed PIN lesions, which were also much larger than those in Pten $^{+/-}$mice and occurred in all lobes (Figure 3, PTEN $^{+/-} ; \mathrm{SOX} 9$ ). As expected, these lesions had high levels of Flag-SOX9, Ki67, and phospho-AKT (Supplemental Figure 3C), and high AR expression as well (Supplemental Figure 3D). Lesions in 2 PTEN $^{+-}$; SOX9 mice had a loss of basal cells (indicated by a lack of high molecular weight cytokeratin staining) and marked host inflammatory reactions, suggesting local invasion (Figure 3, bottom panels).

The SOX 9 transgene in these mice is controlled by the tetracycline operon. Its expression in prostate was stimulated by the tetracycline transactivator (tTA), which is controlled by the androgen-regulated MMTV promoter. Since tTA activity can be repressed by the addition of tetracycline or doxycycline (tetoff), we also addressed whether established PIN lesions would respond to tTA repression by doxycycline. A series of approximately 8-month-old PTEN ${ }^{+-}$;SOX9 mice were treated with doxycycline (added to their food and drinking water) for 2 weeks prior to sacrifice, and PIN lesions in their prostates were compared with untreated controls. There was a decrease in the number of PIN lesions and a decrease in the proportion of larger PIN lesions $\left(>0.5 \mathrm{~mm}^{2}\right.$ ) from $22 \%$ to $9 \%$ in the doxycycline-treated mice (Supplemental Figure 3, E and F), suggesting a continued role for SOX9 in established lesions. 


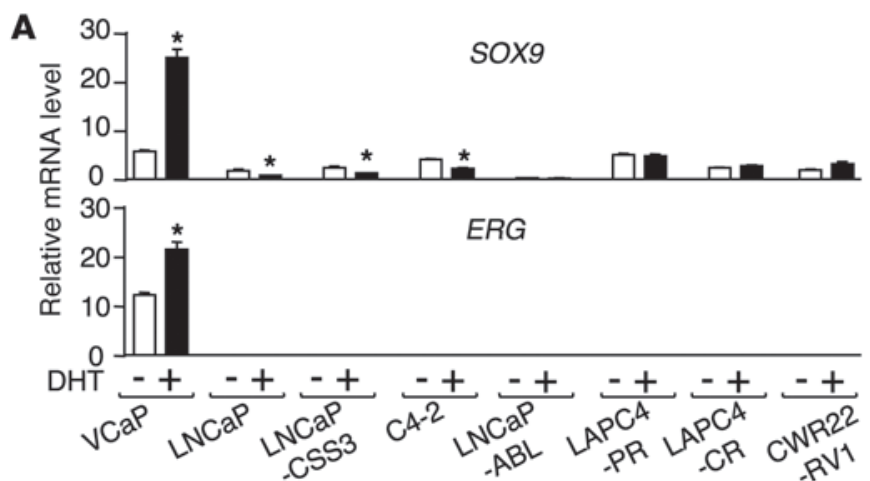

C

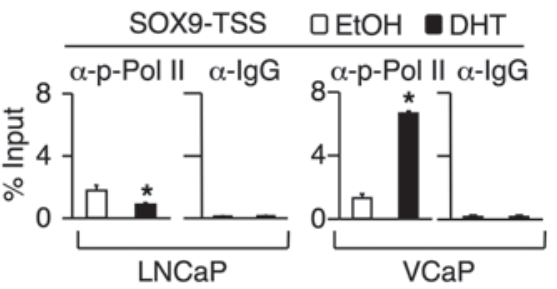

F Androgen-dependent
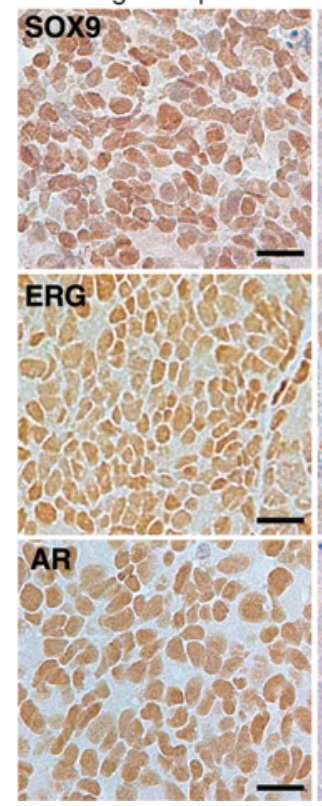

Castrated (4d)

Castration-resistant
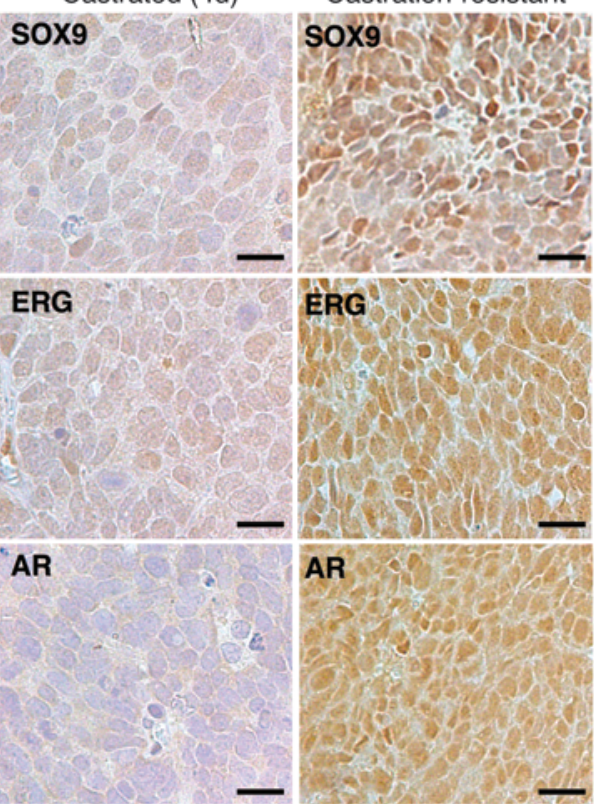

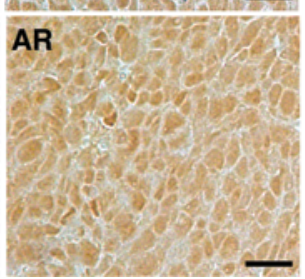

D

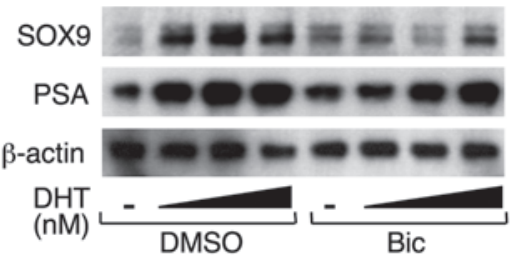

B

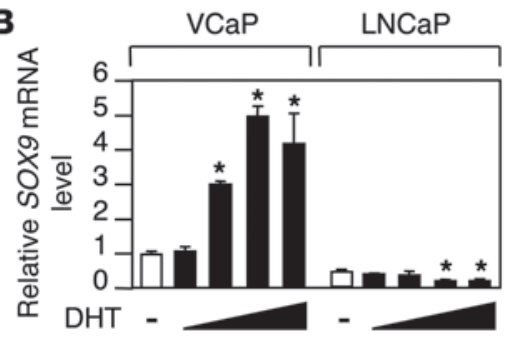

E SOX9 $=-=$ PSA $\mathbb{2} \mathrm{OPO}$ $\beta$-tub DHT -
G

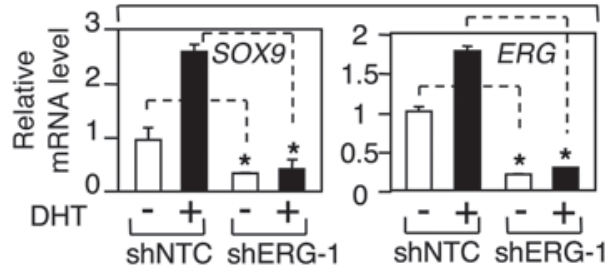

H

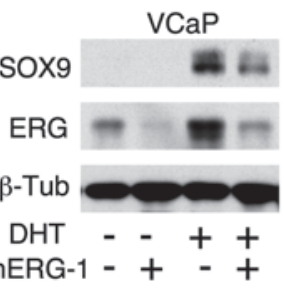

Figure 2

SOX9 expression is androgen and ERG regulated in TMPRSS2:ERG-positive VCaP PCa cells. (A) VCaP; LNCaP; LNCaP-CSS3 (derived from LNCaP cells adapted to hormone-depleted conditions); C4-2; LNCaP-ABL; LAPC4-PR; LAPC4-CR (derived from androgen-dependent or castration-resistant LAPC4 xenografts); and CWR22-Rv1 cells were treated with or without DHT for 24 hours followed by qRT-PCR. (B) VCaP and LNCaP cells were treated with $0,0.01,0.1,1$, or $10 \mathrm{nM} \mathrm{DHT}$ for 24 hours and SOX9 mRNA was measured by qRT-PCR. (C) LNCaP and VCaP cells were stimulated with or without DHT for 4 hours, and binding of activated phospho-RNA polymerase II (phospho-Ser5 on CTD) to the SOX9 transcriptional start site (TSS) was assessed by ChIP. (D) VCaP cells were treated with 0-10 nM (0, 0.1, 1, or 10) $\mathrm{DHT}$ and with vehicle (DMSO) or $10 \mu \mathrm{M}$ Bic for 24 hours and immunoblotted. (E) LNCaP cells were treated with $0,0.01,0.1,1$, or $10 \mathrm{nM}$ DHT for 24 hours, followed by immunoblotting. (F) Expression of SOX9, ERG, or AR was examined by IHC in VCaP xenografts prior to castration (Androgen-dependent), at 4 days after castration, or at relapse (Castration-resistant). Original magnification, $\times 400$; Scale bars: $20 \mu \mathrm{m}$. (G and $\mathbf{H}$ ) VCaP cells stably infected with nontarget control (NTC) or ERG shRNA-1 were treated with or without DHT for 24 hours, and SOX9 or ERG mRNA was then measured by qRT-PCR, or SOX9, ERG, and $\beta$-tubulin protein were assessed by immunoblotting. Data in bar graphs represent means \pm SD of at least 3 biological repeats. Significant differences from DHT-negative controls are indicated $\left(^{\star}\right)$. 


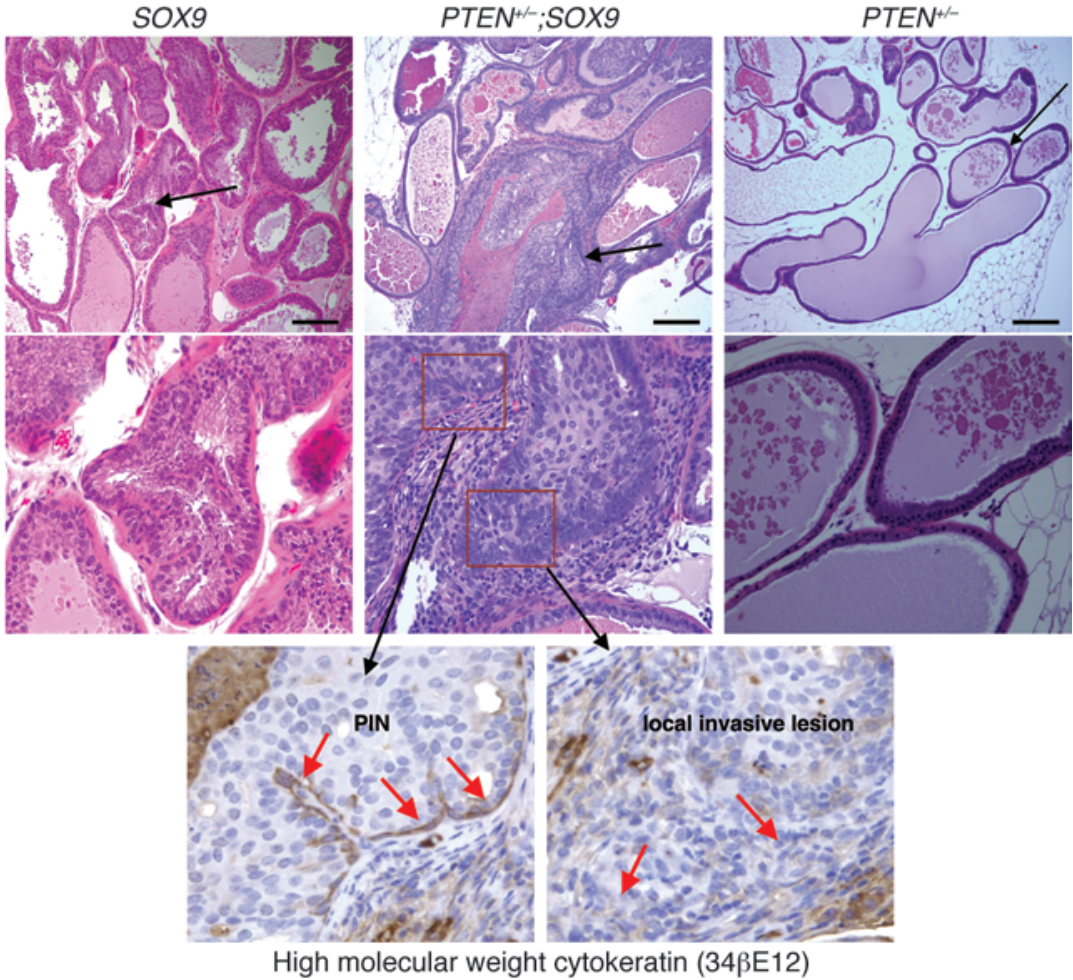

\section{Figure 3}

SOX9 mediates increased proliferation and neoplasia in mouse prostate. Prostates from 8-month-old transgenic mice with the MMTV-driven Flag epitopetagged human SOX9 in prostate epithelium on a Pten intact background (left panels) or on a Pten ${ }^{+/-}$ background (middle panels) were examined. Arrows indicate areas of PIN (left panel) and an area that appears to be focal invasion (middle panel) shown under higher power in the lower panels (stained for high molecular weight cytokeratin, 34ßE12). Lower-power original magnification, $\times 100$; higher-power original magnification, $\times 400$. Scale bars: $200 \mu \mathrm{m}$. Red arrows indicate remaining basal cells in PIN lesions (lower left panel) or the area of local invasion (lower right panel). Right panels show normal histology in age-matched $\mathrm{Pten}^{+/-}$mouse ventral prostate.
We next used Matrigel invasion assays to determine whether SOX9 contributes to ERG-mediated invasion. Consistent with our previous results in LNCaP xenograft cells overexpressing SOX9, which showed increased invasion in vivo (35), SOX9 induction in LNCaP cells with doxycycline-inducible SOX9 (LN-toSOX9, protein induction shown in Supplemental Figure 4A) strongly stimulated basal Matrigel invasion (Figure 4A). DHT-stimulated invasion was not significantly enhanced, which could reflect SOX9 activation of DHT-regulated genes in mediating invasion or alternative mechanisms. To determine whether SOX9 could mediate ERG-dependent invasion, we generated $\mathrm{VCaP}$ cells with doxycycline-inducible SOX9 (VCaP-toSOX9, protein induction shown in Supplemental Figure 4B) and used siRNA to downregulate endogenous ERG (Figure 4B). As expected, Matrigel invasion was decreased by ERG siRNA, which also decreased endogenous SOX9 (Figure 4, B and C). Importantly, invasion was restored by the addition of doxycycline to stimulate exogenous SOX9 expression. Interestingly, while SOX9 induction could restore invasion after ERG knockdown, it did not stimulate invasion in androgen-depleted VCaP cells, indicating that additional DHT-regulated genes in these cells are required to support invasion (Supplemental Figure 4C).

To determine whether VCaP invasion was dependent on endogenous SOX9, we next used a SOX9 shRNA lentivirus (shSOX9-1) to downregulate SOX9 (Figure 4D), which resulted in a marked decrease in DHT-stimulated Matrigel invasion (Figure 4E). This result was confirmed using an independent SOX9 shRNA lentivirus (shSOX9-2) (Supplemental Figure 4, D and E). Finally, a previous study had found that tissue-type plasminogen activator (PLAT), which may contribute to invasion, was the major gene downregulated in response to ERG RNAi in VCaP cells (6). Therefore, we addressed whether PLAT expression was SOX9 regulated. As shown in Figure 4F, SOX9 siRNA markedly decreased the expression of PLAT mRNA in VCaP cells. Moreover, in VCaP cells with doxycycline-regulated SOX9, we found that SOX9 induction with doxycycline could substantially restore PLAT mRNA in cells treated with ERG siRNA (Figure 4G). Together, these results indicate that SOX9 is an effector of ERG-mediated PCa invasion.

To determine whether SOX9 contributes to tumor growth, we next assessed the effects of SOX9 RNAi on growth in vitro and in vivo. In LNCaP cells expressing low levels of SOX9, growth in vitro was not impaired by SOX9 siRNA (Supplemental Figure $4 \mathrm{~F})$. In contrast, infection of $\mathrm{VCaP}$ cells with the shSOX9-1 lentivirus, which markedly downregulates SOX9 protein (Figure 4D), substantially decreased in vitro growth (Figure 4H). Indeed, we have not been able to obtain adequate numbers of these cells for xenograft studies. Infection with the shSOX9-2 lentivirus did not as effectively deplete SOX9 (Supplemental Figure 4D), and in vitro growth was not substantially decreased (not shown). Nonetheless, SOX9 downregulation by shSOX-2 markedly impaired the ability to develop xenografts (Figure 4I). Moreover, in the two shSOX9-2 xenografts that developed, the rate of proliferation (assessed by Ki67 immunostaining) was decreased compared with the control tumor on the opposite flank of the same mouse (Figure 4J).

While these results show that VCaP growth in vitro can be decreased by SOX9 shRNA, we and others have not found marked effects of ERG RNAi on VCaP growth in vitro (5). This may reflect a need to more dramatically deplete SOX9 in order to affect growth versus invasion in vitro, as $\mathrm{VCaP}$ growth in vitro was impaired only by the more efficient shSOX9-1. Consistent with this hypothesis, doxycycline induction of SOX9 can partially rescue VCaP-toSOX9 cells from the growth-suppressive effects of androgen-deprivation VCaP-toSOX9 (which reduces ERG and markedly reduces endogenous SOX9) (Supplemental Figure 4G). As expected, DHT is more effective, as it clearly stimulates the 
A

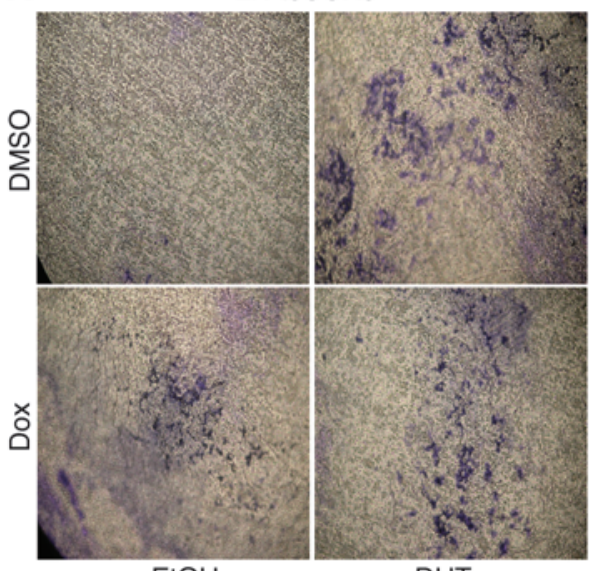

$\mathrm{EtOH}$

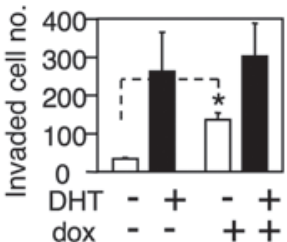

B

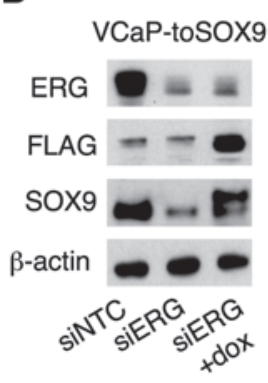

C
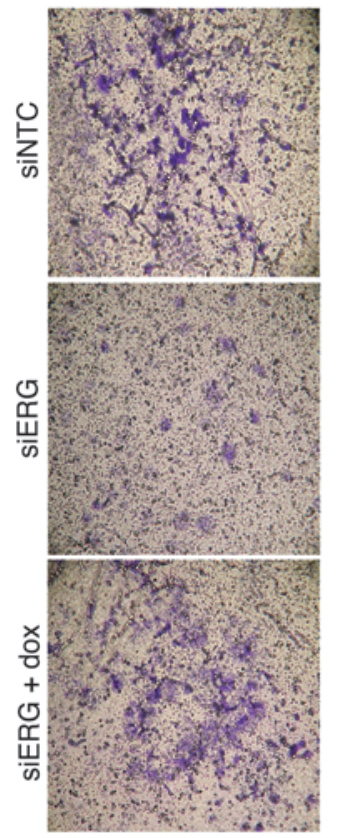

VCaP-tosox9 with DHT

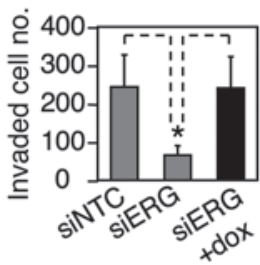

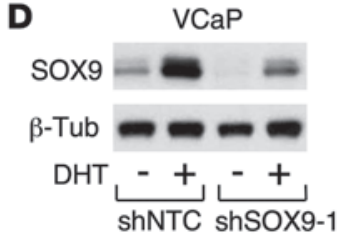

D $\beta$-Tub shNTC shSOX9-1
E

部

$\mathrm{EtOH}$
$\mathrm{VCaP}$

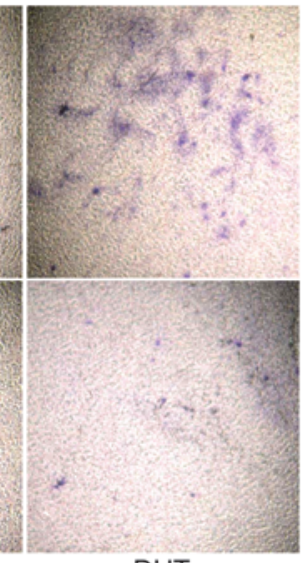

DHT

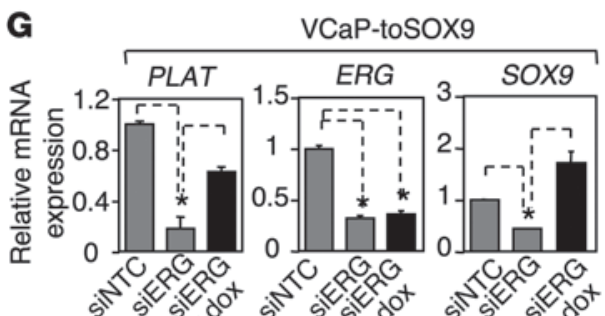

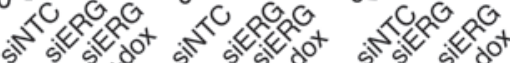

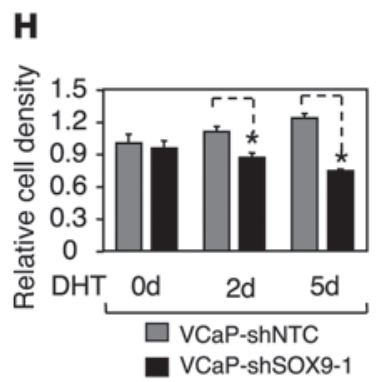

$\mathbf{F}$

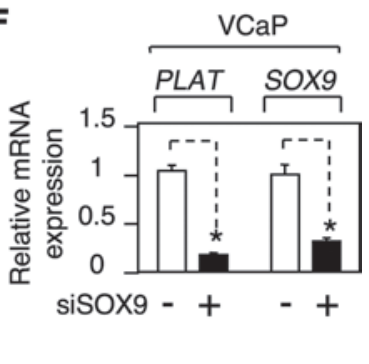

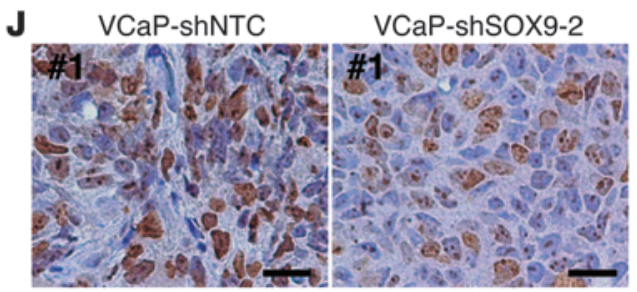

Ki67: $53 \pm 6 \%$

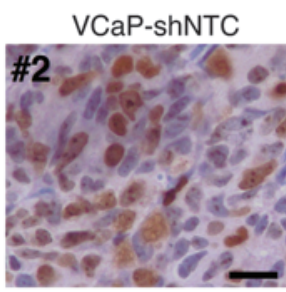

Ki67: $48 \pm 7 \%$

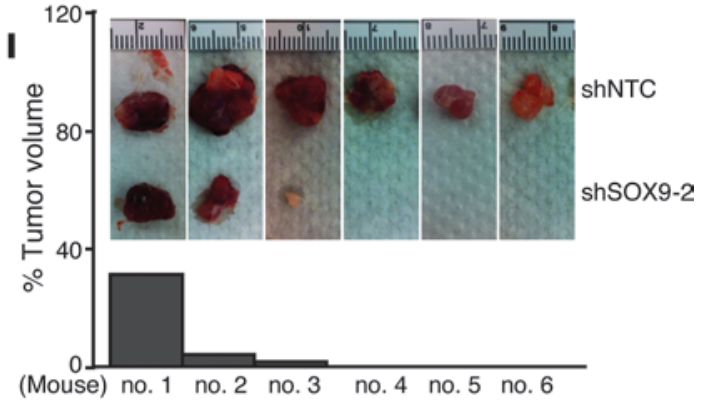




\section{Figure 4}

SOX9 is a major downstream effector of ERG in TMPRSS2:ERG fusion-positive PCa cells. (A) LN-toSOX9 cells treated with DHT and/ or doxycycline (inducing SOX9 expression) were assessed for Matrigel invasion. Cells invading through membrane were stained and quantified. Images are from a representative experiment, and graph represents the mean and SD from 3 independent experiments. (B) VCaP-toSOX9 cells (grown in DHT-supplemented medium) treated with NTC siRNA, ERG siRNA, or ERG siRNA plus doxycycline were immunoblotted as indicated and (C) assessed for invasion. (D) VCaP cells with SOX9 shRNA or control shRNA (shNTC) were treated with DHT and immunoblotted or (E) assessed for invasion. (F) Effect of SOX9 siRNA on PLAT mRNA expression in VCaP cells cultured in FBS medium. (G) VCaP-toSOX9 cells (grown in DHT-supplemented tetracycline-free medium) treated with nontarget control siRNA, ERG siRNA, or ERG siRNA plus doxycycline were analyzed by qRT-PCR. (H) VCaP-shSOX9-1 versus control VCaP-shNTC cells were treated with $\mathrm{DHT}$ for 0,2 , or 5 days, and cell recovery was measured by MTT assay. (I) The same number of VCaP-shNTC or VCaP-shSOX9-2 cells were injected into the left or right flank of the same mouse. When the VCaP-shNTC xenografts reached approximately $1 \mathrm{~cm}$ in diameter ( 8 weeks), the mice were sacrificed and tumor volumes were compared. Graph shows volume of each VCaP-shSOX9 tumor relative to the corresponding control on the opposite flank. (J) IHC for Ki67 in xenograft set numbers 1 and 2 (\%Ki67+ cells based on 4 random areas). Original magnification, $\times 400$. Scale bars: $20 \mu \mathrm{m}$. Data in bar graphs represent means \pm SD of at least 3 biological repeats. dox, doxycycline.

expression of multiple other genes in addition to ERG and SOX9. Taken together, these in vitro and in vivo results further support the conclusion that SOX9 is a critical mediator of ERG effects in TMPRSS2:ERG fusion-positive PCa.

AR directly stimulates SOX9 expression in TMPRSS2:ERG fusion-positive cells. Since ERG expression in TMPRSS2:ERG fusion-positive PCa cells is stimulated by androgen, we initially presumed that the DHT-stimulated increase in SOX9 in VCaP cells was secondary to the increased expression of ERG (Figure 2). However, the rapid induction of $\mathrm{SOX} 9 \mathrm{mRNA}$ and $\mathrm{SOX} 9$ protein in response to DHT suggested that AR may be directly inducing SOX9 (Figure 5, A and B). To determine whether the DHT-stimulated increase in SOX9 mRNA was mediated by increased ERG protein, we used cycloheximide (CHX) to block new protein synthesis. Importantly, the addition of CHX did not prevent the DHT-stimulated increase in SOX9 mRNA (Figure 5C). As a control for the inhibition of protein synthesis, we confirmed that CHX caused a rapid decrease in SOX9 protein in DHT-stimulated and control cells (Supplemental Figure 5A). Moreover, we found that overexpression of full-length ERG or ERG(del1-44), which is expressed in VCaP cells, did not increase SOX9 in AR-negative PC3 cells (Supplemental Figure 5B). These results indicate that AR may be directly stimulating SOX9 expression.

Significantly, examination of available AR ChIP massively parallel DNA sequencing (ChIP-seq) data $(12,44,45)$ for AR binding sites within $50 \mathrm{~kb}$ of the SOX9 gene transcriptional start site revealed a major peak located at approximately $35 \mathrm{~kb} 3^{\prime}$ of the SOX9 gene in VCaP cells that was not present in LNCaP cells (S2 site, Figure 5D and Supplemental Figure 5C). Conversely, the major SOX9 gene-associated AR binding peak in LNCaP cells (S1

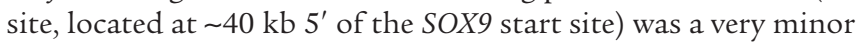
peak in VCaP cells. Moreover, the S1 peak in LNCaP cells was much weaker than the S2 peak in VCaP cells (Figure 5D). Interestingly, a portion of the $\mathrm{S} 1$ site is highly conserved across 17 vertebrate species (Supplemental Figure 5C), although we do not know if this is related to AR function at this site. Using AR ChIP and quantitative PCR ( $\mathrm{qPCR}$ ) with $\mathrm{S} 2$ site-specific primers, we confirmed the marked DHT-stimulated recruitment of AR to this site in VCaP cells (Figure 5E). This could be blocked by the AR antagonist Bic, which, as reported previously, can more weakly stimulate AR binding in the absence of androgen. In contrast, there was minimal DHT-stimulated AR binding to the S2a and S2b sites flanking the S2 site, consistent with the major sharp AR peak observed by ChIPseq (Supplemental Figure 5, C and D).

AR binding to the $\mathrm{S} 1$ and $\mathrm{S} 2$ sites in $\mathrm{VCaP}$ versus $\mathrm{LNCaP}$ cells is compared in detail in Figure 5, F and G, respectively. Weak AR binding to the $\mathrm{S} 1$ site, which could be modestly increased by DHT, was observed in both VCaP and LNCaP cells. Anti-FOXA1 ChIP showed that FOXA1, which has been previously identified as a pioneer transcription factor that is required to initially open loci for subsequent AR binding (46), was bound to the S1 sites in both cells. Low levels of GATA2, which also associates with AR on many AR-regulated genes (46), were present in the LNCaP cells, but not in the VCaP cells. Most importantly, DHT did not stimulate recruitment of the p300 coactivator to the S1 site in either cell, and modestly decreased p300 binding to the S1 site in LNCaP cells. These findings indicate that the weak AR binding to the S1 site does not stimulate SOX9 expression, but may contribute to DHT-mediated SOX9 repression in LNCaP cells.

In contrast to the $\mathrm{S} 1$ site, $\mathrm{AR}$ binding to the $\mathrm{S} 2$ site was dramatically increased by DHT in VCaP cells, but not in LNCaP cells (Figure 5, F and G, middle panels). Since the AR in LNCaP cells has a point mutation in the ligand binding domain (T877A), we also examined a stable LNCaP cell line expressing a 3xFlag-tagged wildtype AR and found that it was similarly recruited to the S1 site by DHT, but not to the S2 site (data not shown). Consistent with the S2 site functioning as an AR-regulated enhancer in VCaP cells and interacting with the promoter, DHT stimulated an association between the S2 site and phospho-RNA polymerase II (Figure 5F, middle panel). FOXA1 binding and DHT-stimulated GATA2 binding to the $\mathrm{S} 2$ site were also observed in the VCaP cells, but not in the LNCaP cells. Moreover, DHT stimulated the recruitment of p300 to the S2 site in VCaP cells. The DHT-stimulated binding of AR, the association with phospho-RNA polymerase II, and the recruitment of p300 at the S2 site in VCaP cells were comparable to those observed for the major AR-regulated enhancer in the PSA gene in both VCaP and LNCaP cells (Figure 5, F and G, bottom panels). Finally, using ChIP we found that levels of monomethylated and dimethylated $\mathrm{H} 3 \mathrm{~K} 4$ (H3K4me1 and $\mathrm{H} 3 \mathrm{~K} 4 \mathrm{me} 2)$, which are associated with active enhancers, were higher at the S2 site in VCaP cells compared with the S1 site in VCaP cells, and were higher than the levels at the S1 and S2 sites in LNCaP cells (Supplemental Figure 5E). Together, these findings indicate that the S2 site is an AR-regulated enhancer for the SOX9 gene in VCaP cells, but not in LNCaP cells.

To assess AR binding to the S2 site in vivo, we performed AR ChIP on VCaP xenografts prior to castration and at 4 days after castration. Comparable levels of AR were bound to the S2 site and to the PSA gene enhancer prior to castration, and AR binding to both sites was markedly decreased after castration (Supplemental Figure 5F). To evaluate the contribution of the S2 site to SOX9 expression in CRPC, we also examined a cell line (VCS2) generated from a castration-resistant VCaP xenograft (45). Basal levels (in steroid-depleted medium) of both PSA and 
A

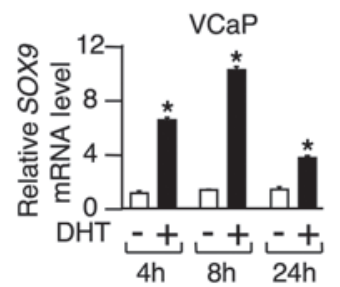

B

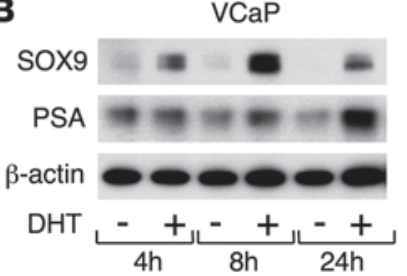

D

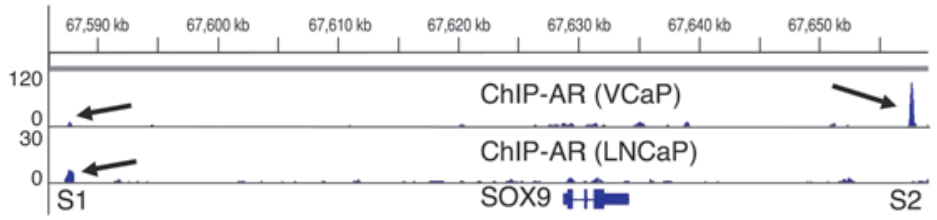

$\mathbf{F}$

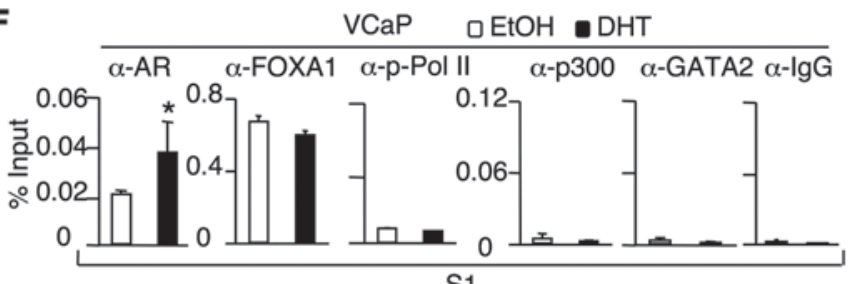

$\alpha$-AR $\quad \alpha$-FOXA1 $\alpha$-p-Pol II $\quad \alpha$-p300 $\alpha$-GATA2 $\alpha$-lgG

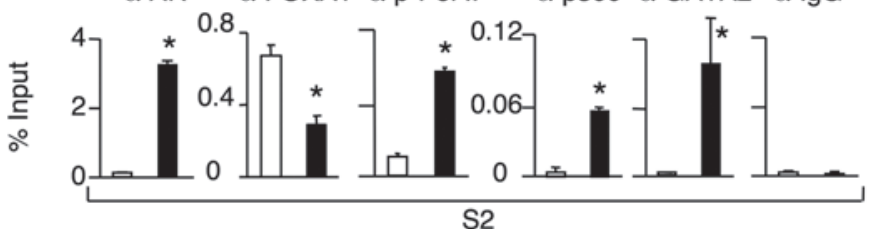

$\alpha$-AR $\quad \alpha$-FOXA1 $\alpha$-p-Pol II $\quad \alpha$-p300 $\alpha$-GATA2 $\alpha$-lgG

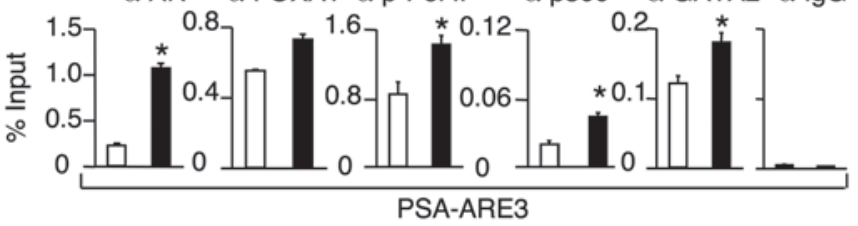

C

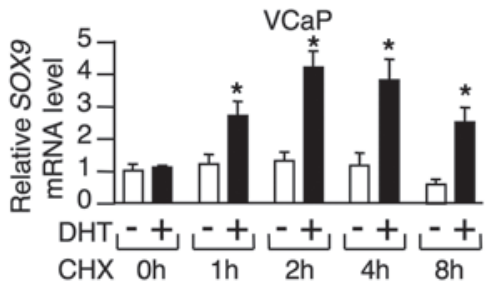

E

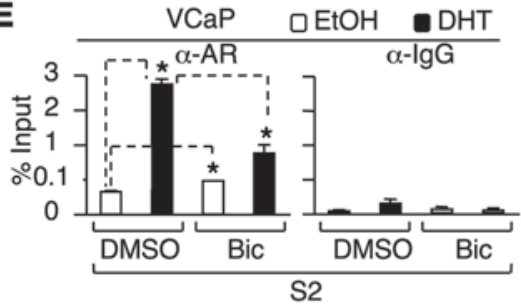

G
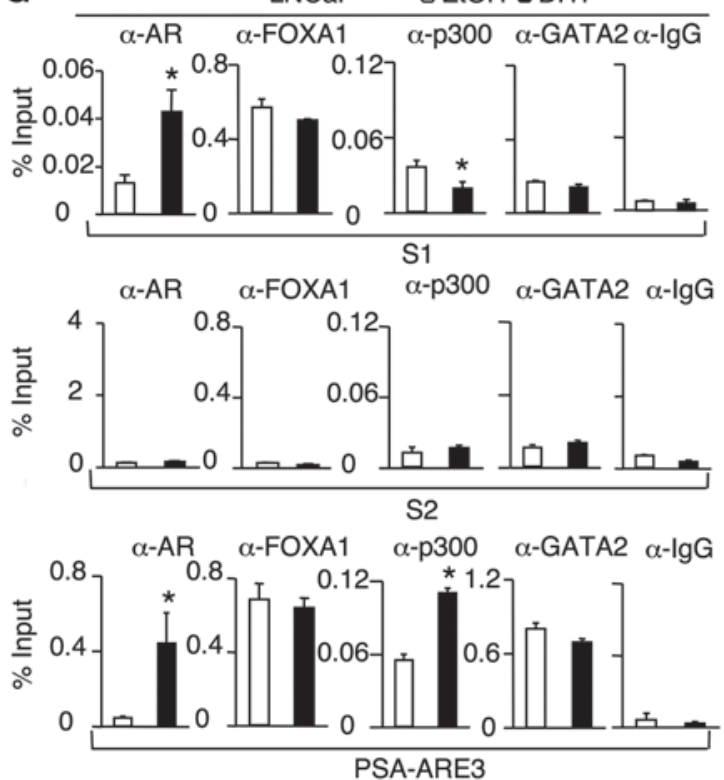

Figure 5

AR directly regulates SOX9 in VCaP cells through activation of an enhancer in the SOX9 gene. (A) VCaP cells were treated with or without DHT for 4,8 , or 24 hours and SOX9 mRNA was measured by GRT-PCR. (B) VCaP cells were treated with or without DHT for 4, 8, or 24 hours and SOX9, PSA, or $\beta$-actin was immunoblotted. (C) VCaP cells were treated with cycloheximide $(10 \mathrm{ng} / \mathrm{mL})$ and DHT or vehicle (ethanol), and SOX9 mRNA was then measured by qRT-PCR after 0-8 hours. (D) Distinct AR binding sites identified by ChIP-seq and ChIP-chip in LNCaP cells (S1 site) and $\mathrm{VCaP}$ cells (S2). A peak at $\mathrm{S} 1$ in $\mathrm{VCaP}$ could also be detected by decreasing the threshold of peak analysis, suggesting that AR binds weakly to this site in VCaP cells. (E) VCaP cells were treated with or without DHT and with $10 \mu \mathrm{M}$ Bic or vehicle control (DMSO) for 4 hours followed by AR ChIP and qPCR for the S2 site. (F) VCaP cells were treated with or without DHT for 4 hours followed by AR, FOXA1, active RNA polymerase II (phospho-Ser5 on CTD), p300, or GATA2 ChIP and qPCR for the S1 site, S2 site, or PSA enhancer ARE (PSA-ARE3). (G) LNCaP cells were treated with or without DHT for 4 hours followed by AR, FOXA1, p300, or GATA2 ChIP and QPCR for the S1 site, S2 site, or PSA enhancer. The DHT concentration used in experiments was $10 \mathrm{nM}$. Data in bar graphs represent means $\pm \mathrm{SD}$ of at least 3 biological repeats.

SOX9 mRNA were higher in the VCS2 cells compared with the parental VCaP cells, and could be further elevated by DHT and suppressed by Bic (Supplemental Figure 5G). Basal p300 and GATA2 binding on the PSA enhancer in VCS2 cells was very high, consistent with the substantial basal PSA expression in these cells (Supplemental Figure 5H, bottom panels). Similarly to VCaP cells, DHT in the VCS2 cells markedly induced AR, GATA2, and p300 binding to the S2 site, but not to the S1 site
(Supplemental Figure 5H). These findings further support the conclusion that the S2 site is an AR-regulated enhancer for the SOX9 gene in TMPRSS2:ERG fusion-positive PCa.

ERG activates the AR-regulated S2 enhancer in TMPRSS2:ERG fusion-positive PCa. We next examined available ERG ChIP-seq data in $\mathrm{VCaP}$ cells to determine whether ERG may be regulating AR binding to the S2 site (12). Significantly, we found a broad peak of ERG binding over approximately $2 \mathrm{~kb}$ that overlapped 
A

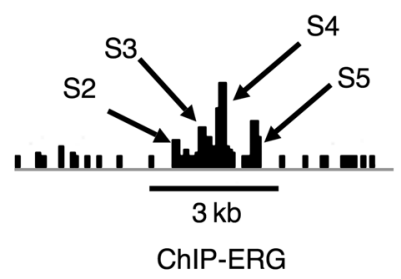

B

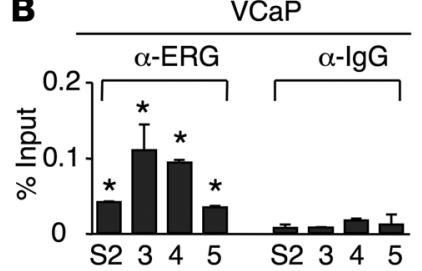

C

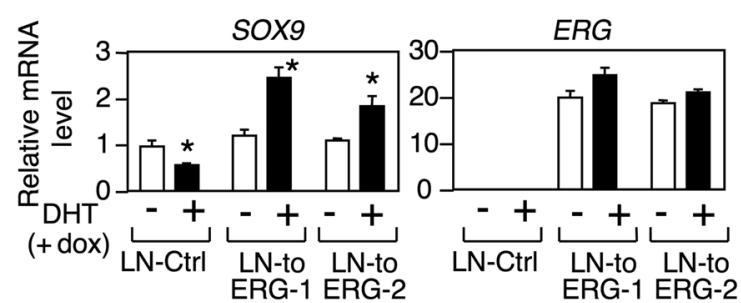

E

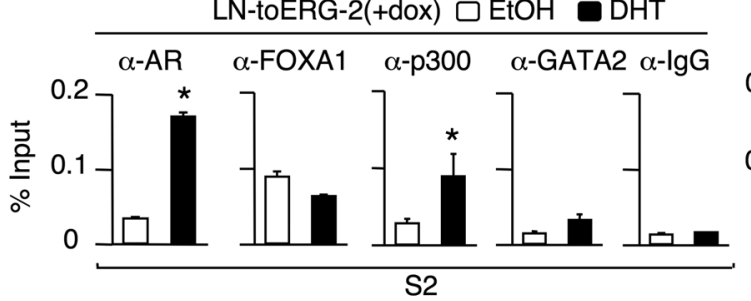

D
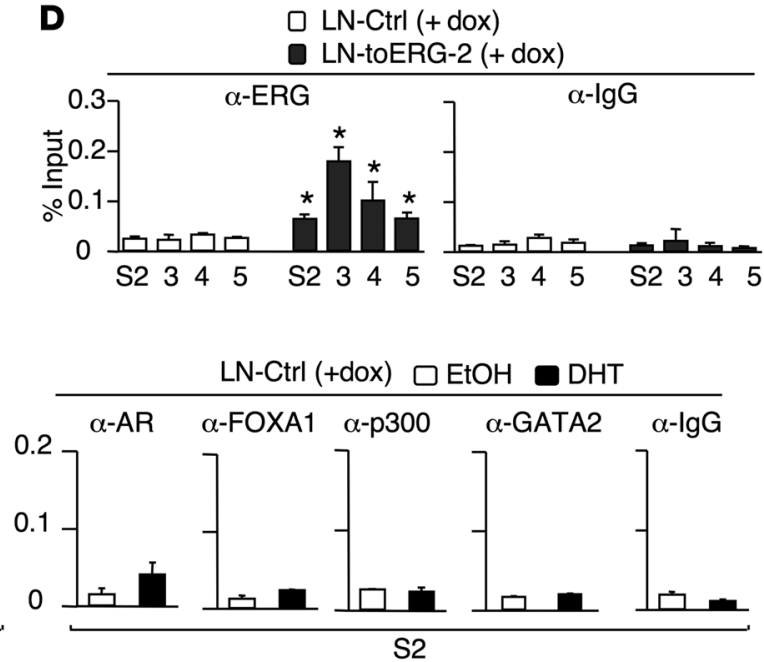

Figure 6

ERG activates AR-regulated S2 site enhancer in TMPRSS2:ERG fusion-positive PCa. (A) ERG binding site adjacent to and overlapping the S2 site. (B) Anti-ERG ChIP confirmed ERG binding to this region (S2, S3, S4, and S5) in VCaP cells. (C) Control LNCaP parental cells (LN-Ctrl) or independently derived LNCaP cell lines with doxycycline-inducible ERG expression (LN-toERG-1 or LN-toERG-2) were pretreated with doxycycline (+ dox) for 3 days and then treated with or without $10 \mathrm{nM}$ DHT for 24 hours. SOX9 or ERG mRNA was examined by qRT-PCR. (D) Anti-ERG ChIP for ERG binding on the S2 region (S2-5) in ERG-expressing LNCaP cells (LN-toERG-2) versus parental LNCaP cells. (E) LN-toERG-2 or LNCaP-Ctrl cells were treated with or without $10 \mathrm{nM}$ DHT for 4 hours followed by AR, FOXA1, p300, or GATA2 ChIP and qPCR for the S2 site. The DHT concentration used in experiments was $10 \mathrm{nM}$. Data in bar graphs represent means \pm SD of at least 3 biological repeats.

the S2 site (Figure 6A). Using anti-ERG ChIP and qPCR with a series of primers targeting S2 and its neighboring sites (S3-S5) that cover this approximately $2-\mathrm{kb}$ span, we confirmed ERG binding to this region (Figure 6B). We next used ERG siRNA to determine whether ERG downregulation altered AR binding to the S2 site in VCaP cells. Importantly, although we could greatly reduce cellular ERG protein levels, ChIP studies showed that ERG binding over the S2-S5 region was reduced but not eliminated (data not shown). Nonetheless, ERG siRNA reduced DHT-stimulated AR binding to the S2 site (Supplemental Figure $6 \mathrm{~A})$, and similarly reduced DHT-stimulated expression of SOX9 (Supplemental Figure 6B).

As a complimentary approach, we determined whether the ERG(del1-44) expressed from the TMPRSS2:ERG fusion in VCaP cells is sufficient to confer DHT stimulation of SOX9 expression in fusion-negative LNCaP cells. In 2 independent LNCaP cell lines expressing a doxycycline-inducible ERG(del1-44) protein (LN-toERG-1 and -2), we found that DHT could stimulate SOX9 mRNA expression (Figure 6C) and protein expression (Supplemental Figure 6C), which could be blocked by Bic (Supplemental Figure 6D). Importantly, this exogenous ERG in LNCaP cells was bound to the S2-S5 region identified in the VCaP cells (Figure 6D). Moreover, the S2 site in the ERG-expressing LNCaP cells had increased basal FOXA1 binding prior to DHT stimulation, consistent with ERG binding to this site and making it accessible to transcription factors (Figure 6E). Finally, DHT strongly stimulated recruitment of AR and p300 to the previously unavailable S2 site in the ERG-expressing LNCaP cells (Figure 6E). It should be noted that LNCaP cells have a distinct gene fusion that results in androgen-stimulated overexpression of another ETS factor, ETV1, indicating that not all ETS factors can stimulate SOX9 expression. Together, these findings demonstrate that ERG increases SOX9 expression in TMPRSS2:ERG fusion-positive PCa cells by opening an AR-regulated enhancer in the SOX9 gene.

Identification of additional ERG-dependent AR-regulated genes in TMPRSS2:ERG fusion-positive PCa. Since these data indicated that ERG was functioning as a pioneer factor for the AR-regulated S2 site $\operatorname{SOX} 9$ gene enhancer, we hypothesized that ERG expression may open cryptic AR-regulated enhancers associated with additional genes and thereby further reprogram AR transcription in TMPRSS2:ERG fusion-positive PCa. As outlined below, to test this hypothesis we first sought to identify additional genes (exemplified by SOX9) that were androgen stimulated and contained unique AR binding sites in VCaP cells compared with LNCaP cells. We then assessed whether the unique AR binding sites in these genes were associated with ERG binding sites. For this analysis, we used expression microarray data from androgen-stimulated VCaP cells and LNCaP cells $(44,45)$. In addition, we used 
A

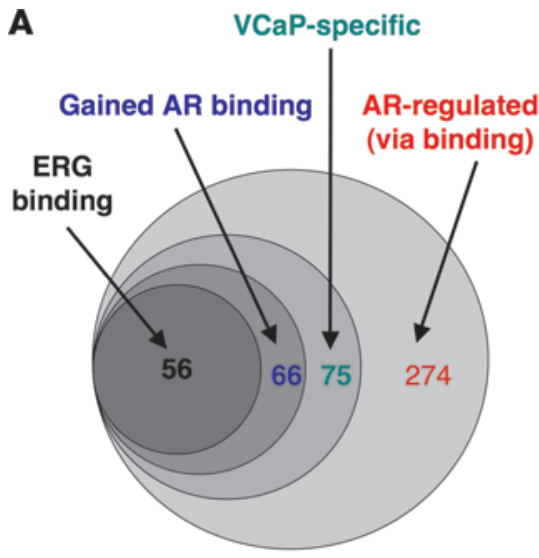

(471 AR-activated genes)
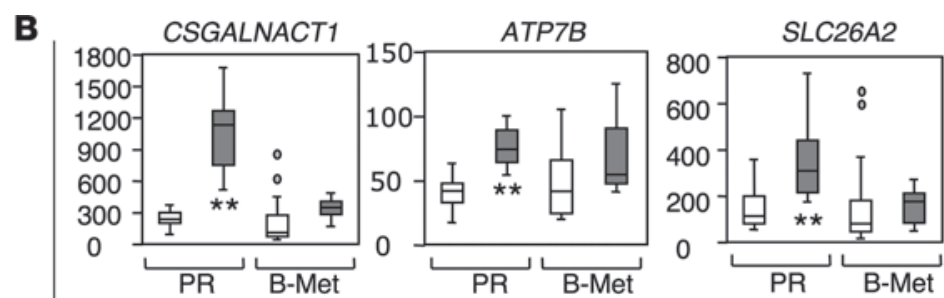

$\square$ Fusion-negative a Fusion-positive
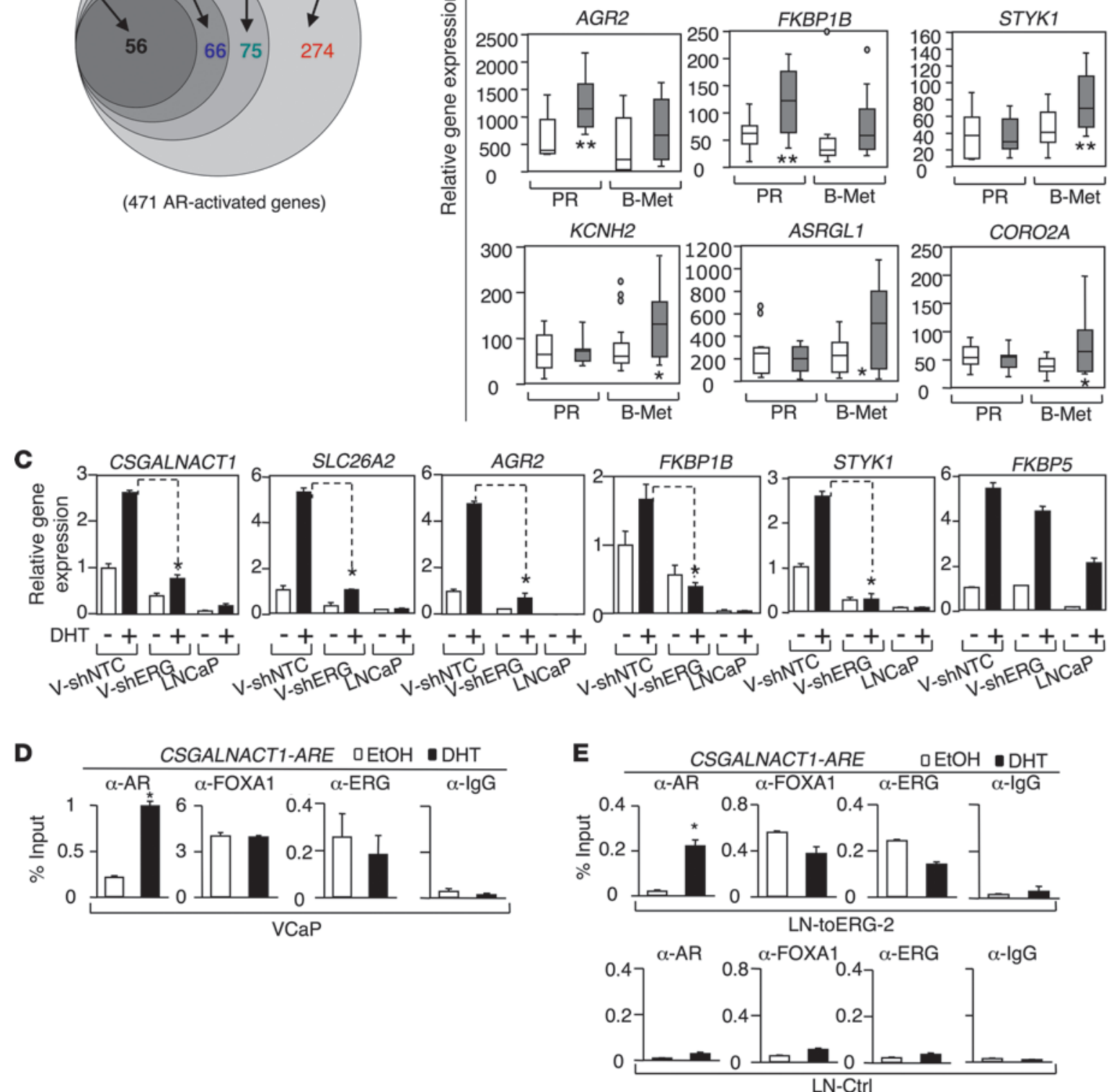

\section{Figure 7}

Identification of additional direct AR-regulated genes associated with TMPRSS2:ERG fusion. (A) Identification of genes that are DHT induced with AR binding sites in VCaP cells, but not in LNCaP cells, and have associated ERG binding sites. (B) Quartile plots from Affymetrix arrays showing expression of 9 genes from this subset in fusion-positive versus fusion-negative primary PCa (PR) or CRPC bone metastases (B-Met). Significant differences are indicated. (C) Five genes were further selected for qRT-PCR analysis in DHT-treated (24 hours) VCaP cells stably expressing an NTC shRNA (V-shNTC), ERG shRNA-1 (V-shERG), or LNCaP cells. Androgen stimulation of the control FKBP5 gene, which is strongly androgen regulated in LNCaP and VCaP cells, was not significantly altered in the V-shERG versus control V-shNTC VCaP cells. (D and E) AR, FOXA1, or ERG binding on the CSGALNACT1-ARE (Supplemental Figure 7C) in VCaP, LN-toERG-2 (with dox), or LNCaP-Ctrl (with dox) cells was measured by ChIP-qPCR. 


\section{Table 1}

List of ERG-dependent AR-activated genes

\begin{tabular}{lll}
\hline AGR2 & GAS2L1 & PPIB \\
ANXA2 & GIPC1 & PPM1B \\
ASRGL1 & GNE & RABAC1 \\
ATP7B & HMGCS2 & RAP1GAP \\
CDK2AP2 & KCNH2 & SCRG1 \\
CMTM8 & KCTD3 & SLC1A5 \\
CORO2A & STYK1 & SLC26A2 \\
CSGALNACT1 & KRT19 & SLC27A3 \\
DPP3 & LAT2 & SLC2A10 \\
EPDR1 & LIPG & SOX9 \\
FAM13C & METRNL & TESC \\
FKBP1B & MYOF & TMEM8 \\
FXYD3 & OCEL1 &
\end{tabular}

VCaP-specific AR-regulated genes with ERG binding site located within $4 \mathrm{~kb}$ of the AR binding site.

published ChIP microarray technology (ChIP-chip) and ChIP-seq data identifying AR binding sites in DHT-treated LNCaP and VCaP cells, and ERG binding sites in VCaP cells $(12,44,45)$.

Using the $\mathrm{VCaP}$ microarray expression data, we initially identified 471 genes as being androgen stimulated (greater than 2-fold, AR-activated genes) in VCaP cells (Figure 7A). Among these 471 genes, the VCaP AR ChIP-seq data showed that 274 (58\%) had AR binding sites within $50 \mathrm{~kb}$ of their transcription start sites (versus a background of $37 \%$ of all genes in these cells having an AR binding site within $50 \mathrm{~kb}$ ), consistent with them being directly regulated by AR (Supplemental Figure 7A). Of these 274 genes that appeared to be AR regulated in $\mathrm{VCaP}$ cells, analysis of the microarray expression data in LNCaP cells showed that 75 genes had less than a $50 \%$ expression level in $\mathrm{LNCaP}$ cells versus $\mathrm{VCaP}$ cells, and were not DHT induced in LNCaP cells (less than 1.5-fold induction). Comparison of the AR ChIP-chip and ChIP-seq data in LNCaP cells versus VCaP cells then showed that 66 of these 75 genes had AR binding sites in $\mathrm{VCaP}$ cells that were not present in $\mathrm{LNCaP}$ cells.

Significantly, using the VCaP ERG ChIP-seq data, we then found that ERG binding sites were located within $20 \mathrm{~kb}$ of the AR site in 56 of these 66 genes (Figure 7A). Forty of these 66 genes (61\%) have ERG binding sites within $1 \mathrm{~kb}$ of the AR binding sites. By comparison, among the total set of $274 \mathrm{AR}-$ regulated genes in $\mathrm{VCaP}$ cells, only 97 genes (35\%) have ERG binding sites within $1 \mathrm{~kb}$ of the AR binding sites, indicating that these VCaP-specific AR binding sites are further enriched for ERG binding. The VCaP-specific AR-regulated genes with closely associated ERG and AR binding sites (within $4 \mathrm{~kb}$ ) are listed in Table 1.

Gene ontology analysis of these genes revealed only a weak overall association with secretion (Supplemental Figure 7B). To identify genes in this group that may be clinically significant, we assessed their expression in TMPRSS2:ERG fusion-positive versus TMPRSS2:ERG fusion-negative primary PCa and CRPC bone metastases (40). In addition to SOX9 (Figure 1), 9 of these genes were increased in TMPRSS2:ERG fusion-positive PCa primary tumors (PR) or bone marrow metastases (B-Met) (Figure 7B). AR and ERG binding sites for these 9 genes are shown in Supplemental Figure 7C. However, these genes were also not strongly associated with specific pathways (Supplemental Figure 7B, right panel), and none were significantly increased in both the primary and metastatic CRPC tumors.
Three of these 9 genes (CSGALNACT1, ATP7B, and SLC26A2) were increased in the fusion-positive primary tumors from the MSKCC dataset (Supplemental Figure 7D) (41).

To confirm the ERG-dependent androgen regulation of these genes, we selected 5 of the 9 and assessed the effects of ERG shRNA on their DHT-stimulated expression in VCaP cells. In all cases, qRT-PCR showed low expression in LNCaP cells and confirmed that basal and DHT-stimulated expression in VCaP cells was markedly diminished by ERG shRNA, while expression of a gene that is strongly androgen stimulated in $\mathrm{VCaP}$ and $\mathrm{LNCaP}$ cells (FKBP5) was not markedly altered (Figure 7C). Since CSGALNACT1 expression was strongly correlated with TMPRSS2:ERG fusion in both clinical datasets, we further assessed DHT-stimulated recruitment of transcription factors to its AR binding site in VCaP and LNCaP cells. As observed for SOX9, FOXA1 was constitutively associated with the CSGALNACT1 AR binding site in VCaP cells (Figure 7D) and in LNCaP cells stably expressing ERG (LN-toERG-2, Figure 7E), as well as DHT-stimulated AR binding to this site, while neither FOXA1 nor AR were bound to this site in control LNCaP cells (Figure 7E). Finally, DHT strongly stimulated CSGALNACT1 mRNA expression in LNCaP cells expressing ERG, but not in control LNCaP cells, and this expression was blocked by Bic (Supplemental Figure 7E). Overall, this genome-wide study shows that ERG allows AR to bind and transactivate a series of genes in TMPRSS2:ERG fusion-positive PCa that are not AR regulated in fusion-negative tumors.

\section{Discussion}

Approximately half of PCa cases express the AR-driven TMPRSS2:ERG-fusion gene, but the downstream targets of ERG that contribute to PCa remain to be established. We found that increased SOX9 expression was correlated with TMPRSS2:ERG fusion in 3 independent PCa cohorts, and confirmed the ERG-dependent expression of SOX9 in TMPRSS2:ERG-positive VCaP cells. We further demonstrated that ERG regulates SOX9 indirectly by functioning as a pioneer factor to open a cryptic AR-regulated enhancer in the SOX9 gene, and that ERG can similarly redirect AR to a series of other genes. SOX9 expression in fetal prostate is required for ductal morphogenesis, and its aberrant expression has been linked to a number of cancers including PCa. We confirmed that SOX9, similarly to ERG, stimulates tumor invasion and can drive neoplasia when overexpressed in mouse prostate. Moreover, we showed that SOX9 RNAi impairs ERG-mediated tumor invasion and suppresses tumor growth in vitro and in vivo. Taken together, these studies reveal a molecular mechanism through which ERG functions to redirect AR to a series of genes including $S O X 9$, and show that SOX9 is a major downstream effector of both AR and ERG in TMPRSS2:ERG fusion-positive PCa.

Significantly, primary PCa is characterized by increased formation of glands that lack basal cells and may have well-formed lumens (Gleason pattern 3) or ill-defined lumens (Gleason pattern 4), which is very reminiscent of developing prostate. Therefore, we further suggest that one function of aberrant ERG and AR-driven SOX9 expression is to reactivate the prostate ductal morphogenesis program that SOX9 normally regulates during prostate development. However, additional functions of SOX9 related to the maintenance of stem/progenitor pools are also likely to contribute to PCa development or progression. Finally, it should be emphasized that the functions of SOX9 expressed in adult prostate basal cells are not known, but SOX9 may contribute to the expression 
of paracrine factors that support the luminal epithelium. In this case, ERG and AR-mediated SOX9 expression in PIN lesions may stimulate the autocrine production of these factors, and thereby permit the loss of basal cells that is a defining feature of PCa. Further studies are clearly needed to define the precise sets of SOX9-regulated genes that contribute to fetal prostate development, adult basal cell functions, and PCa.

Although these data reveal that ERG opens a cryptic ARregulated enhancer in the SOX9 gene, the precise mechanisms through which this occurs and the roles of other proteins including FOXA1 remain to be precisely defined. A straightforward model is that ERG binding directly or indirectly increases local H3K4 methylation and subsequent binding of FOXA1, which then facilitates androgen-stimulated binding of AR. However, while FOXA1 can interact with AR and functions as a pioneer factor for AR binding to other enhancers, further RNAi studies are needed to determine whether AR binding to this site is dependent on FOXA1 or is facilitated by interactions with other proteins including ERG. Further studies are also needed to characterize the interaction between the S2 site and the SOX9 promoter, and specifically to determine whether the interaction is mediated by an AR-stimulated chromatin looping mechanism, as has been shown for other AR-regulated enhancers.

Substantial overlap between AR and ERG binding sites was also observed in a recent study. However, that study found that ERG was functioning to repress AR transcriptional activity at these sites, as well as to directly decrease expression of the $A R$ gene (12). Our data are not inconsistent with this previous study, as we have not examined the effects of ERG on genes that are normally strongly stimulated by androgens. Moreover, they are not inconsistent with the hypothesis that ERG may stimulate tumor growth in part by repressing AR activity on a subset of ARregulated genes. However, while this mechanism may contribute to tumor progression, we suggest that the ERG-mediated redirection of $A R$, rather than the suppression of $A R$, makes a more significant contribution to tumor initiation in TMPRSS2:ERG fusion-positive PCa.

In contrast to PCa cells expressing ERG, androgen repressed the low basal expression of SOX9 in LNCaP cells. Consistent with this result, a previous study found that castration increased SOX9 expression in murine prostate epithelium (36). Our data indicate that AR binding to the S1 site may mediate this repression, but more studies are needed to determine the role of this site in the response to androgen. In any case, the evolutionary conservation of the S1 site suggests a significant role for this region in regulating SOX9 expression. Indeed, it should be emphasized that additional mechanisms may drive SOX9 expression in TMPRSS2:ERG fusion-negative PCa, and may further modulate its expression in fusion-positive PCa. Interestingly, among these other mechanisms are the $\mathrm{Wnt} / \beta$-catenin and receptor tyrosine kinase/MAP kinase pathways $(24,29,47)$. Therefore, higher levels of SOX9 expression (above the AR- and ERG-stimulated levels in fusion-positive tumors or above the lower basal levels in fusion-negative tumors) may be a biomarker for the activation of additional pathways that can drive $\mathrm{PCa}$ progression through SOX9-dependent and -independent mechanisms. Further studies of mechanisms controlling SOX9 and identification of the downstream genes it regulates should provide new therapeutic targets, which may be particularly relevant to TMPRSS2:ERG fusion-positive PCa.

\section{Methods}

Cell culture and xenografts. LNCaP cells were cultured in RPMI-1640 with $10 \%$ FBS. VCaP cells were cultured in DMEM with $10 \%$ FBS, and VCS2 cells were cultured in DMEM with $8 \%$ charcoal-dextran-stripped serum (CSS) and 2\% FBS (HyClone). The LN-toERG cell lines were generated by transfecting the pTET-Splice/ERG vector into a LNCaP clone carrying the pcDNA6/TR, followed by selection (34). PTET-Splice/ERG vector was made by inserting the HA-del 1-44 ERG coding fragment between the Sal1SpeI sites of the pTET-Splice vector. The VCaP-toSOX9 cell line was generated by infection of lentivirus generated with the pLVX-Tet-On Advanced system (Clontech, in which a 3xFlag-SOX9 fragment was cloned between the BamHI and EcoRI sites of pLVX-Tight-Puro vector). The tet-inducible cells were maintained in RPMI-1640 with $10 \%$ tetracycline-free FBS. For androgen stimulation assays, cells were grown to $50 \%-60 \%$ confluence in medium with $5 \%$ CSS for 3 days prior to treatment. Cells were stimulated with $10 \mathrm{nM}$ DHT unless otherwise indicated. VCaP xenografts were established in the flanks of male SCID mice by injecting approximately 2 million cells in 50\% Matrigel.

RT-PCR, immunoblotting, and IHC. Quantitative real-time RT-PCR amplification was performed with Taqman 1-step RT-PCR reagents and results were normalized to coamplified 18S RNA or GAPDH. Data shown are representative of at least 3 experiments. Primers and probes are listed in Supplemental Materials. Proteins were extracted by boiling for 15 minutes in $2 \%$ SDS and detected by blotting with anti-ERG (Santa Cruz Biotechnology), anti-PSA (BioDesign), anti-AR (Upstate USA), anti-SOX9 (34), anti- $\beta$-actin (Abcam), or anti- $\beta$-tubulin (Upstate USA). Gels shown are representative of at least 3 independent experiments. Paraffin sections underwent antigen retrieval and were then blocked using $5 \%$ goat serum and avidin-blocking solution (Vector Laboratories). Anti-AR, anti-ERG (Santa Cruz Biotechnology), anti-SOX9, anti-FLAG (Sigma-Aldrich), or anti-Ki67 (Dako) were then added overnight at $4{ }^{\circ} \mathrm{C}$, followed by biotinylated secondary antibody and streptavidin-HRP (Vector Laboratories). Sections compared in each figure were stained at the same time and photographed under identical conditions.

SOX9 IHC and ERG fusion status in PCa tissue microarrays. IHC was performed on tissue microarrays (TMAs) created from prostatectomy samples among men with prostate cancer in the Physicians' Health and Health Professionals Follow-up Studies tumor cohort as described in detail in 2 recent publications $(42,48)$. IHC was performed on 5 -micron sections of 6 TMAs using the SOX9 antibody as described above. The immunostained TMAs were then scanned and quantitatively scored using the Ariol SL-50 system (Genetix, Molecular Devices), after the areas of tumor were identified and electronically circled by a study pathologist (X. Yuan). Scores were generated using the MultiStain assay, providing areas of staining per cell (area_score), intensity of staining (intensity_score), and a combination of the two to produce a logarithmic score with an approximately normal distribution (addscore). The TMPRSS2:ERG fusion status of the TMA samples was determined by IHC using the ERG antibody (43). A 2-tailed Student's $t$ test was used to compare mean SOX9 expression in fusion-positive and -negative PCa.

ChIP. Dispensed cells or xenograft tissues were formalin fixed, lysed, and sonicated to break the chromatin into 500-800 bp fragments. AntiAR, anti-p300, anti-OCT1, anti-GATA2, anti-RNA polymerase II, rabbit or mouse IgG (Santa Cruz Biotechnology), anti-RNA polymerase II CTD repeat (phospho Ser5), or anti-FOXA1 (Abcam) were used to precipitate chromatin fragments from cell extracts. Real-time qPCR was used to analyze binding to the SOX9-TSS, S1-S5 sites, and androgen-responsive elements (AREs) in the PSA enhancer (ARE3) or TMPRSS2 enhancer (14 kb upstream). The primers are listed in the Supplemental Information. We used real-time qPCR (SYBR Green) to amplify the DNA fragment in the 
antibody-precipitated DNA and the unprecipitated input DNA to calculate $\Delta C T$ values. The relative quantity $(R Q)$ value $\left(R Q=2^{-\Delta C T}\right)$ represented the precipitated DNA relative to input. Results are represented as mean \pm STD for replicate samples. Data are representative of at least 3 experiments.

ChIP-seq. ChIP-seq assay was performed in VCaP cells treated with DHT for 4 hours. ChIP-seq library construction for AR was performed as previously described (44), and the libraries were sequenced to $35 \mathrm{bp}$ with the Illumina Genome Analyzer. Significantly $\left(P<1 \times 10^{-15}\right)$ enriched regions were detected with MACS software using default parameters.

Cell proliferation and invasion assays. Cell growth was examined using the MTT assay (Sigma-Aldrich). Cell invasion was measured using the Cell Invasive Assay Kit from Chemicon following the manufacturer's protocol. Briefly, cell suspensions in an upper chamber containing 800,000 cells/ $\mathrm{ml}$ in serum-free medium were treated with or without $10 \mathrm{nM} \mathrm{DHT}$ and then monitored for invasion into a lower chamber containing RPMI1640 medium with $10 \% \mathrm{FBS}$. After 72 hours of incubation at $37^{\circ} \mathrm{C}$, cells attached to the membrane of the lower chamber were stained.

Transgenic mice. A 3xFlag-SOX9 cDNA fragment was cloned into the Hind III-EcoRV site of the pTet-Splice vector, which contains 7 copies of the tet operator (TetO; Invitrogen). To generate TetO-SOX9 transgenic mice, the Xho I-Not I fragment containing the TetO-3xFlag-SOX9 and SV40 intron/ polyadenylation signal was gel purified and microinjected into the pronuclei of fertilized mouse eggs at the Beth Israel Deaconess Transgenic Core Facility. The MMTV-tTA mice (49) were provided by M. Kelliher (University of Massachusetts Medical School, Worcester, Massachusetts, USA). The $\mathrm{Pten}^{+/-}$mice (C57Bl/6 background) were provided by P. Pandolfi (50). The combined Pten $^{+/-}$transgenic mice overexpressing SOX9 were on a mixed $\mathrm{C} 57 \mathrm{Bl} / 6$ and $\mathrm{FVB}$ background.

Data deposits. The following have been deposited in the Gene Expression Omnibus database: PCa tissue Affymetrix gene expression microarray data (Gene Expression Omnibus accession number GSE32269); VCaP cell line
Affymetrix gene expression data (accession number GSE32344); and the results of the ChIP-seq analysis for AR binding in VCaP cells (accession number GSE32345).

Statistics. Data in bar graphs represent means \pm SD of at least 3 biological repeats. Statistical analysis was performed by a 2-tailed Student's $t$ test. ${ }^{*} P<0.05 ;{ }^{*} P<0.01$ versus control values. $P<0.05$ was considered statistically significant.

Study approval. The analyses of deidentified human tissue samples for gene expression were approved by the Beth Israel Deaconess Medical Center's IRB. The development and analyses of genetically modified mice described in the manuscript were approved by the IACUC of Beth Israel Deaconess Medical Center.

\section{Acknowledgments}

This work was supported by grants from the NIH (R01 DK079962 to X. Yuan, and K99 CA166507 to C. Cai); SPORE in Prostate Cancer P50 CA090381; postdoctoral awards from the Department of Defense (W81XWH-08-1-0160 to H. Wang, and W81XWH-09-1-0159 to C. Cai); Idea Development Award from the Department of Defense (W81XWH-09-1-0435 to S.P. Balk); and by a Challenge Award from the Prostate Cancer Foundation.

Received for publication August 31, 2012, and accepted in revised form December 17, 2012.

Address correspondence to: Steven P. Balk or Xin Yuan, Beth Israel Deaconess Medical Center, 330 Brookline Avenue, Boston, Massachusetts 02215, USA. Phone: 617.735.2065; Fax: 617.735.2050; E-mail: sbalk@bidmc.harvard.edu (S.P. Balk). Phone: 617.735.2066; Fax: 617.735.2050; E-mail: xyuan@bidmc. harvard.edu (X. Yuan).
1. Lamont KR, Tindall DJ. Androgen regulation of gene expression. Adv Cancer Res. 2010;107:137-162.

2. Tomlins SA, et al. Recurrent fusion of TMPRSS2 and ETS transcription factor genes in prostate cancer. Science. 2005;310(5748):644-648.

3. Kumar-Sinha C, Tomlins SA, Chinnaiyan AM. Recurrent gene fusions in prostate cancer. Nat Rev Cancer. 2008;8(7):497-511.

4. Perner S, et al. TMPRSS2-ERG fusion prostate cancer: an early molecular event associated with invasion. Am J Surg Pathol. 2007;31(6):882-888.

5. Cai C, Wang H, Xu Y, Chen S, Balk SP. Reactivation of androgen receptor-regulated TMPRSS2:ERG gene expression in castration-resistant prostate cancer. Cancer Res. 2009;TMPRSS2(15):6027-6032.

6. Tomlins SA, et al. Role of the TMPRSS2-ERG gene fusion in prostate cancer. Neoplasia. 2008; 10(2):177-188

7. Wang J, Cai Y, Yu W, Ren C, Spencer DM, Ittmann M. Pleiotropic biological activities of alternatively spliced TMPRSS2/ERG fusion gene transcripts. Cancer Res. 2008;68(20):8516-8524.

8. Carver BS, et al. Aberrant ERG expression cooperates with loss of PTEN to promote cancer progression in the prostate. Nat Genet. 2009;41(5):619-624.

9. King JC, et al. Cooperativity of TMPRSS2-ERG with PI3-kinase pathway activation in prostate oncogenesis. Nat Genet. 2009;41(5):524-526.

10. Zong Y, Xin L, Goldstein AS, Lawson DA, Teitell MA, Witte ON. ETS family transcription factors collaborate with alternative signaling pathways to induce carcinoma from adult murine prostate cells. Proc Natl Acad Sci US A. 2009;106(30):12465-12470.

11. Klezovitch O, et al. A causal role for ERG in neoplastic transformation of prostate epithelium. Proc Natl Acad Sci U S A. 2008;105(6):2105-2110.

12. Yu J, et al. An integrated network of androgen recep- tor, polycomb, and TMPRSS2-ERG gene fusions in prostate cancer progression. Cancer Cell. 2010; 17(5):443-454.

13. Kunderfranco P, et al. ETS transcription factors control transcription of EZH2 and epigenetic silencing of the tumor suppressor gene Nkx3.1 in prostate cancer. PLoS One. 2010;5(5):e10547.

14. Gupta S, et al. FZD4 as a mediator of ERG oncogene-induced WNT signaling and epithelial-to-mesenchymal transition in human prostate cancer cells. Cancer Res. 2010;70(17):6735-6745.

15. Wang $J$, et al. Activation of NF-\{kappa\}B by TMPRSS2/ERG fusion isoforms through toll-like receptor-4. Cancer Res. 2011;71(4):1325-1333.

16. Schepers GE, Teasdale RD, Koopman P. Twenty pairs of sox: extent, homology, and nomenclature of the mouse and human sox transcription factor gene families. Dev Cell. 2002;3(2):167-170.

17. Pritchett J, Athwal V, Roberts N, Hanley NA, Hanley KP. Understanding the role of SOX9 in acquired diseases: lessons from development. Trends $\mathrm{Mol}$ Med. 2011;17(3):166-174.

18. Wagner T, et al. Autosomal sex reversal and campomelic dysplasia are caused by mutations in and around the SRY-related gene SOX9. Cell. 1994;79(6):1111-1120.

19. Foster JW, et al. Campomelic dysplasia and autosomal sex reversal caused by mutations in an SRY-related gene. Nature. 1994;372(6506):525-530.

20. Seymour PA, et al. SOX9 is required for maintenance of the pancreatic progenitor cell pool. Proc Natl Acad Sci U S A. 2007;104(6):1865-1870.

21. Vidal VP, et al. Sox9 is essential for outer root sheath differentiation and the formation of the hair stem cell compartment. Curr Biol. 2005;15(15):1340-1351.

22. Lincoln J, Kist R, Scherer G, Yutzey KE. Sox9 is required for precursor cell expansion and extracellular matrix organization during mouse heart valve development. Dev Biol. 2007;305(1):120-132.

23. Furuyama K, et al. Continuous cell supply from a Sox9-expressing progenitor zone in adult liver, exocrine pancreas and intestine. Nat Genet. 2011;43(1):34-41.

24. Blache P, et al. SOX9 is an intestine crypt transcription factor, is regulated by the Wnt pathway, and represses the CDX2 and MUC2 genes. J Cell Biol. 2004;166(1):37-47.

25. Lu B, et al. Analysis of SOX9 expression in colorectal cancer. Am J Clin Pathol. 2008;130(6):897-904.

26. Vidal VP, Ortonne N, Schedl A. SOX9 expression is a general marker of basal cell carcinoma and adnexal-related neoplasms. J Cutan Pathol. 2008;35(4):373-379.

27. Jiang SS, et al. Upregulation of SOX9 in lung adenocarcinoma and its involvement in the regulation of cell growth and tumorigenicity. Clin Cancer Res. 2010;16(17):4363-4373.

28. Kordes U, Hagel C. Expression of SOX9 and SOX10 in central neuroepithelial tumor. J Neurooncol. 2006;80(2):151-155.

29. Ling S, et al. An EGFR-ERK-SOX9 signaling cascade links urothelial development and regeneration to cancer. Cancer Res. 2011;71(11):3812-3821.

30. Guo W, et al. Slug and Sox9 cooperatively determine the mammary stem cell state. Cell. 2012;148(5):1015-1028.

31. Matheu A, et al. Oncogenicity of the developmental transcription factor Sox9. Cancer Res. 2012;72(5):1301-1315.

32. Thomsen MK, et al. SOX9 elevation in the prostate promotes proliferation and cooperates with PTEN loss to drive tumor formation. Cancer Res. 2010;70(3):979-987.

33. Schaeffer EM, et al. Androgen-induced programs 
for prostate epithelial growth and invasion arise in embryogenesis and are reactivated in cancer. Oncogene. 2008;27(57):7180-7191.

34. Wang H, McKnight NC, Zhang T, Lu ML, Balk SP, Yuan X. SOX9 is expressed in normal prostate basal cells and regulates androgen receptor expression in prostate cancer cells. Cancer Res. 2007;67(2):528-536.

35. Wang $\mathrm{H}$, et al. SOX9 is expressed in human fetal prostate epithelium and enhances prostate cancer invasion. Cancer Res. 2008;68(6):1625-1630.

36. Thomsen MK, Butler CM, Shen MM, Swain A. Sox9 is required for prostate development. Dev Biol. 2008;316(2):302-311.

37. Acevedo VD, et al. Inducible FGFR-1 activation leads to irreversible prostate adenocarcinoma and an epithelial-to-mesenchymal transition. Cancer Cell. 2007;12(6):559-571

38. Zhang X, Cowper-Sal Lari R, Bailey SD, Moore JH, Lupien M. Integrative functional genomics identifies an enhancer looping to the SOX9 gene disrupted by the $17 \mathrm{q} 24.3$ prostate cancer risk locus. Genome Res. 2012;22(8):1437-1446.
39. Huang Z, et al. Sox9 is required for prostate development and prostate cancer initiation. Oncotarget. 2012;3(6):651-663.

40. Stanbrough M, et al. Increased expression of genes converting adrenal androgens to testosterone in androgen-independent prostate cancer. Cancer Res. 2006;66(5):2815-2825.

41. Taylor BS, et al. Integrative genomic profiling of human prostate cancer. Cancer Cell. 2010;18(1):11-22.

42. Hendrickson WK, et al. Vitamin D receptor protein expression in tumor tissue and prostate cancer progression. J Clin Oncol. 2011;29(17):2378-2385.

43. Pettersson A, et al. The TMPRSS2:ERG rearrangement, ERG expression, and prostate cancer outcomes: a cohort study and meta-analysis. Cancer Epidemiol Biomarkers Prev. 2012;21(9):1497-1509.

44. Wang Q, et al. Androgen receptor regulates a distinct transcription program in androgen-independent prostate cancer. Cell. 2009;138(2):245-256.

45. Cai C, et al. Androgen receptor gene expression in prostate cancer is directly suppressed by the androgen receptor through recruitment of lysine-specific demethylase 1. Cancer Cell. 2011;20(4):457-471

46. Wang Q, et al. A hierarchical network of transcription factors governs androgen receptor-dependent prostate cancer growth. Mol Cell. 2007;27(3):380-392.

47. Murakami S, Kan M, McKeehan WL, de Crombrugghe B. Up-regulation of the chondrogenic Sox9 gene by fibroblast growth factors is mediated by the mitogen-activated protein kinase pathway. Proc Natl Acad Sci U S A. 2000;97(3):1113-1118.

48. Barry M, et al. $\alpha$-Methylacyl-CoA racemase expression and lethal prostate cancer in the Physicians' Health Study and Health Professionals Follow-up Study. Prostate. 2012;72(3):301-306.

49. Hennighausen L, Wall RJ, Tillmann U, Li M, Furth PA. Conditional gene expression in secretory tissues and skin of transgenic mice using the MMTVLTR and the tetracycline responsive system. J Cell Biochem. 1995;59(4):463-472.

50. Di Cristofano A, Pesce B, Cordon-Cardo C, Pandolfi PP. Pten is essential for embryonic development and tumour suppression. Nat Genet. 1998;19(4):348-355. 